\title{
The gravitational collapse of Plummer protostellar clouds
}

\author{
G. Arreaga-García ${ }^{1}$, J. Klapp-Escribano ${ }^{2}$, and F. Gómez-Ramírez ${ }^{2,3}$ \\ ${ }^{1}$ Centro de Investigación en Física, Universidad de Sonora, Blvd. Rosales y Blvd. Encinas s/n Col. Centro, Hermosillo 83000, \\ Sonora, Mexico \\ e-mail: garreaga@cajeme.cifus.uson.mx \\ 2 Instituto Nacional de Investigaciones Nucleares, Carretera Mexico-Toluca s/n, La Marquesa, Ocoyoacac 52750, Estado de Mexico, \\ Mexico \\ e-mail: jaime.klapp@inin.gob.mx \\ 3 Facultad de Ciencias, Universidad Autónoma del Estado de Mexico, El Cerrillo Piedras Blancas 50200, Estado de Mexico, Mexico \\ e-mail: fer_7mx@yahoo.com.mx
}

Received 2 February 2009 / Accepted 22 October 2009

\begin{abstract}
The growing observational evidence that main-sequence and pre-main-sequence protostars are found in binary and multiple systems suggests that they are formed by a fragmentation of collapsing molecular cloud cores. In this paper we present the results of a set of numerical simulations aimed to study the gravitational collapse and fragmentation of a centrally condensed cloud with a nearly flat central density region and a surrounding gas envelope. In order to describe this cloud structure, we use the Plummer radial density profile which satisfies the observed fact that protostellar clouds have a flat central density profile in their innermost region. We consider the cloud to be made of molecular hydrogen and describe the thermodynamics with a single barotropic equation of state, which includes a critical density $\rho_{\text {crit }}$ as a unique free parameter that determines a thermodynamical change on the collapsing gas: from an isothermal to an adiabatic regime. In this paper we consider four different values for the initial radius $R_{\mathrm{c}}$ of the cloud, ranging from 2675 to $19913 \mathrm{AU}$. In the models, for each ratio $R_{\mathrm{c}} / R_{0}$ of the cloud to core radius, we use two critical density values: $\rho_{\text {crit }}=5.0 \times 10^{-14}$ and $5.0 \times 10^{-12} \mathrm{gr} \mathrm{cm}^{-3}$. When the adiabatic change regime starts earlier, we find interesting gas structures as a result of the collapse, although these structures are different according to the initial mass content of the envelope and the initial angular velocity of the cloud. When the thermodynamical change occurs later, i.e., for $\rho_{\text {crit }}=5.0 \times 10^{-12} \mathrm{gr} \mathrm{cm}^{-3}$, we observe that the previously found structure is almost erased to give place instead to a single clump of gas without any adorning spiral arms. In general, we find that as the extension of the envelope mass increases, the possibility of a model to produce a multiple system decreases. This is a result of the initial configuration of our models, namely that with bigger envelopes their cores have a lower ratio of rotational to gravitational energy $\beta_{0}$, a lower ratio of thermal plus rotational to gravitational energy $\alpha_{0}+\beta_{0}$, and a lower angular velocity $\Omega_{0}$, which induces a stronger collapse which in turn contributes to the destruction of the structure that is formed during the initial phases of the collapse. Thus in a sufficient quantity rotational energy is crucial for the fragmentation to occur and survive.
\end{abstract}

Key words. stars: formation - stars: low-mass

\section{Introduction}

The star formation process that occurs in the interstellar medium begins when a prestellar molecular hydrogen cloud becomes gravitationally bound. This earliest stage of cloud evolution lasts $\sim 10^{6}$ years and is completed when a new star system is born within the cloud.

In the central region of the system, a dense core is formed as the prestellar cloud collapses under its own self-gravity. The system is then composed of the central condensed core, usually named the protostellar core, and its surrounding gas envelope.

This protostellar phase is characterized by a high accretion rate onto the protostellar core, so that about $50 \%$ of the original mass located in the surrounding gas envelope falls into the core in about $10^{4}$ years. Eventually, when most of the gas envelope has been accreted or dissipated, the accretion rate becomes significantly lower than in the protostellar stage.

There are uncertainties in the evolution timescale of the cloud because it is obtained from statistical arguments. Despite this, any satisfactory star formation theory must reproduce at least the occurrence of these two stages of evolution, namely the prestellar and protostellar stages, see Ward-Thompson (2002).
We are interested in calculating the dynamical evolution of a cloud with the characteristics described above, although we begin in the protostellar stage without any concerns about the detailed process that the cloud has follow to reach such a stage. What matters most to us is to follow the gravitational collapse in the successful mathematical model of the observed structure of protostellar clouds.

Submillimeter dust emission and near-infrared absorption observations of background stars suggest the mass structure of protostellar clouds is strongly centrally condensed, with a nearly constant central density value of $\approx(3-10) \times 10^{5}$ molecules $\mathrm{cm}^{-3}$ in their innermost region (ranging from a few to $10^{3} \mathrm{AU}$ ). For the outer region, the protostellar surrounding gas envelope has a density profile that falls with the radius as $r^{-\eta}$, with $\eta$ a constant, see Myers (2005); André et al. (2004).

Recently Whitworth \& Ward-Thompson (2001) proposed an empirical model to describe an isolated and static protostellar cloud with a radial density profile given by the Plummer (1911) function. Based on observational lifetimes, these authors constrained the value of the exponent $\eta$ to be no more than $\eta=4$. Then they computed analytically the subsequent evolution of the protostellar cloud with the assumption that the gas has 
negligible pressure and rotation. They concluded that this Plummer model captures some of the observed behavior of the protostellar clouds. For instance, by comparing it to the specific case of the observed $L 1544$ dense core, Tafalla et al. (1998) claimed the Plummer model to fit the observed lifetime and accretion rate well.

The Plummer radial density function includes three free parameters: $\rho_{0}$, establishing the central density value at the onset of the protostellar cloud collapse; a critical radius $R_{0}$ that sets up the end of the approximately constant part of the radial density curve for the innermost matter $\left(0<r \leq R_{0}\right)$; and an exponent $\eta$ that fixes the density fall rate for a large radius $\left(r \gg R_{0}\right)$.

In Arreaga-García et al. $(2007,2008)$ we have furthermore studied the evolution of two types of models according to their initial radial density profile, which is either uniform or Gaussian. The uniform density model has been studied by many groups worldwide using different codes based on grids or particles, see for instance Bodenheimer et al. (2000) and references therein. As we successfully reproduced the uniform model results with a particle-based code, we established the reliability of our calculations. This was important because the Gaussian cloud model has been less studied with particle-based codes than the uniform model, despite the fact that the Gaussian model takes into account the observed property of protostellar clouds, which is that they are strongly centrally condensed.

For the reasons given above, we study in this paper the gravitational collapse of protostellar clouds with the Plummer radial density profile, as proposed by Whitworth \& Ward-Thompson (2001). The models include rotation and pressure as in ArreagaGarcía et al. (2007, 2008).

We will discuss the outcome of the simulations as we change the extension of the gas envelope surrounding the constant part of the Plummer radial density profile as well as the critical density value, after which the collapse changes from isothermal to adiabatic, see Sect. 2.2.

As Arreaga-García et al. (2007, 2008), we use here the fully parallelized Gadget 2 code, which implements the Smoothed Particle Hydrodynamics (SPH) technique for solving the hydrodynamic equations coupled to self-gravity. Once gravity has produced a substantial contraction of the protostellar cloud, the gas begins to heat. In order to take this increase of temperature into account, we use a barotropic equation of state, as was proposed by Boss et al. (2000), to model the thermodynamics of the gas.

The outline of the paper is as follows. In Sect. 2 we describe the initial particle distribution of the simulations: radial density profile, mass perturbation and the energy ratios $\alpha$ and $\beta$. We give the values chosen for some of the Gadget 2 parameters. In Sect. 3 the simulations are presented, including the physical properties of the resulting protostellar cores. In Sect. 4 we discuss the relevance of our simulations in view of reports elsewhere, and finally we make some concluding remarks.

\section{Initial conditions and computational considerations}

\subsection{Initial density profile}

In Eq. (1) we show the mathematical function in which we are interested. It describes the observed fact that protostellar clouds have a flat central density profile in their innermost region. It was first introduced by Plummer (1911) and later on studied by
Whitworth \& Ward-Thompson (2001) in the context of the star formation theory. It reads as follows:

$\rho(r)=\rho_{0}\left(\frac{R_{0}}{\sqrt{r^{2}+R_{0}^{2}}}\right)^{\eta}$,

where $\rho_{0}, R_{0}$ and $\eta$ are the three free parameters that fix the shape of the curve. The main characteristic of this Plummer-like function is its radial density, constant at first and then rapidly falling off with the radius increasing to the outermost regions. Plummer (1911) showed the usefulness of a radial density function of this kind to model the matter distribution of globular clusters.

In order to apply the Plummer density function to model the specific case of the observed $L 1544$ protostellar dense core, Whitworth \& Ward-Thompson (2001) fixed the free parameters to the following values:

$$
\begin{aligned}
& \rho_{0}=3.0 \times 10^{-18} \mathrm{grcm}^{-3}=8.96 \times 10^{5} \text { molecules } \mathrm{cm}^{-3}, \\
& R_{0}=8.06 \times 10^{16} \mathrm{~cm}=0.026 \mathrm{pc}, \\
& \eta=4 .
\end{aligned}
$$

The mass contained in this core is $M_{0}=2.81 \times 10^{33} \mathrm{gr} \equiv$ $1.42 M_{\odot}$, and for the density $\rho_{0}$ the free fall timescale $t_{\mathrm{ffc}}$ is 38460 years, which is the same for all models. Following Whitworth \& Ward-Thompson (2001), we use these same parameter values and keep them fixed for all our simulations.

We have accomplished to have a set of 10 million SPH particles for all our simulations represent the initial configuration of the protostellar cloud model. For instance, in Fig. 1 we show the initial density profile as it is numerically measured for different cloud radii $R_{\mathrm{c}}$ for the four initial configurations IC0, IC1, IC2 and IC3, see Sect. 2.5 for more details of the initial configurations and models. The average density $\rho_{\mathrm{av}}$ for each initial configuration is shown in Table 1 and in Fig. 2 we plot the total mass contained in the protostellar cloud as a function of the radius $r$, as it is obtained by direct integration of the Plummer function.

\subsection{Equation of state}

According to astronomical observations, the regions from which stars are formed consist basically of molecular hydrogen clouds at a temperature of $\sim 10 \mathrm{~K}$. Therefore, the ideal equation of state is a good approximation to account for the thermodynamics of the gas in these clouds. However, once gravity has produced a substantial contraction of the cloud, the opacity increases, the collapse changes from isothermal to adiabatic and the gas begins to heat. To include this increase in temperature into our calculations, we use the barotropic equation of state proposed by Boss et al. (2000).

In order to correctly describe the non-isothermal regime, one needs to solve the radiative transfer problem coupled to the hydrodynamic equations, including a fully self-consistent energy equation to obtain a precise knowledge of the dependence of temperature on density. The implementation of radiative transfer has already been included in some mesh-based codes. In SPH, the incorporation of radiative transfer has in general not been very satisfactory, perhaps with the exception of Whitehouse \& Bate (2006), in which they used the flux-limited diffusion approximation to model the collapse of molecular cloud cores. These authors suggested that there are important differences in 
Table 1. Parameters of the collapse models and outcome of the simulations.

\begin{tabular}{|c|c|c|c|c|c|c|c|c|}
\hline Initial configuration & Model & $\frac{R_{\mathrm{c}}}{R_{0}}$ & $\begin{array}{c}\rho_{\mathrm{av}} \\
\mathrm{grcm}^{-3}\end{array}$ & $\begin{array}{c}M_{\mathrm{c}} \\
\mathrm{gr}\end{array}$ & $\begin{array}{c}\Omega_{\mathrm{c}} \\
\times 10^{-13} \mathrm{rad} / \mathrm{s}\end{array}$ & $\begin{array}{c}c_{0} \\
\mathrm{~cm} / \mathrm{s}\end{array}$ & $\begin{array}{c}\rho_{\text {crit }} \\
\mathrm{gr} \mathrm{cm}^{-3}\end{array}$ & Outcome \\
\hline IC0 & A0 & 0.5 & $2.23 \times 10^{-18}$ & $6.28 \times 10^{32}$ & 5.80 & 10620.35 & $5.0 \times 10^{-14}$ & triple \\
\hline IC0 & B0 & & & & & & $5.0 \times 10^{-12}$ & filament \\
\hline IC 1 & A1 & 1.5 & $6.95 \times 10^{-19}$ & $5.14 \times 10^{33}$ & 3.90 & 18751.51 & $5.0 \times 10^{-14}$ & quadruple \\
\hline IC1 & B1 & & & & & & $5.0 \times 10^{-12}$ & single \\
\hline $\mathrm{IC} 2$ & $\mathrm{~A} 2$ & 2.5 & $2.43 \times 10^{-19}$ & $8.34 \times 10^{33}$ & 2.95 & 19942.80 & $5.0 \times 10^{-14}$ & binary \\
\hline $\mathrm{IC} 2$ & B2 & & & & & & $5.0 \times 10^{-12}$ & single \\
\hline IC3 & A3 & 3.7 & $9.22 \times 10^{-20}$ & $1.04 \times 10^{34}$ & 2.22 & 20344.14 & $5.0 \times 10^{-14}$ & binary \\
\hline IC3 & B3 & & & & & & $5.0 \times 10^{-12}$ & single \\
\hline
\end{tabular}
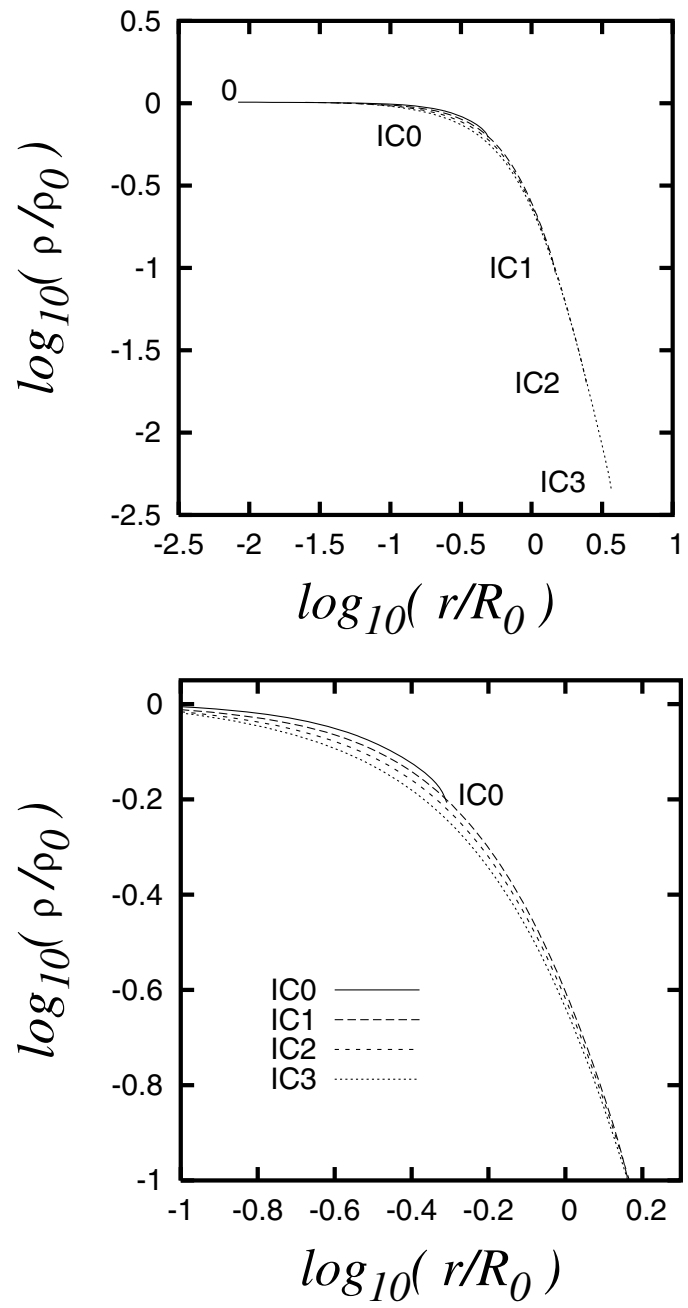

Fig. 1. Initial radial density profile for the initial configurations ICO, IC1, IC2, and IC 3 with $R_{0}=8.06 \times 10^{16} \mathrm{~cm}$ and $\rho_{0}=3.0 \times 10^{-18} \mathrm{gr} \mathrm{cm}^{-3}$. The lower panel is an amplification of the top panel. It should be noted that the profile curves have different radial extension according to the initial configuration. The label along each curve has been placed at its point of maximum radial extension.

the temperature evolution of the cloud when radiative transfer is properly taken into account.

However, after comparing the results of our simulations in Arreaga-García et al. $(2007,2008)$ with those of Whitehouse \& Bate (2006) for the uniform density cloud, we concluded that the barotropic equation of state in general behaves quite well and that we can capture the essential dynamical behavior of the

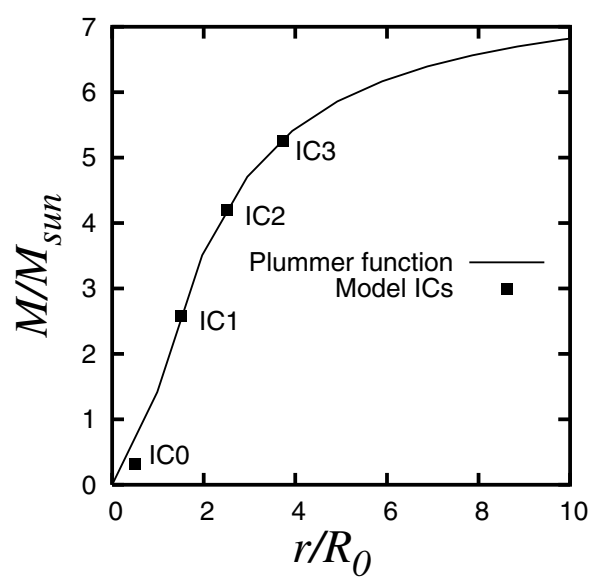

Fig. 2. Mass contained in a Plummer protostellar cloud as a function of the radius. We calculate the mass from direct integration of the Plummer function (solid line) and by counting the SPH particles of the initial configuration (black squares).

collapse. The simulations in this paper are consequently carried out using the following barotropic equation of state:

$p=c_{0}^{2} \rho\left[1+\left(\frac{\rho}{\rho_{\text {crit }}}\right)^{\gamma-1}\right]$,

where $\rho_{\text {crit }}$ defines the critical density above which the collapse changes from isothermal to adiabatic, and for a molecular hydrogen gas the ratio of specific heats $\gamma \equiv 5 / 3$, because we only consider translational degrees of freedom.

In order to study the effect of changing the critical density, we consider the following two values:

$\rho_{\text {crit }}=\left\{\begin{array}{l}5.0 \times 10^{-14} \\ 5.0 \times 10^{-12} \mathrm{gr} \mathrm{cm}^{-3} .\end{array}\right.$

The critical density values $\rho_{\text {crit }}$ that we have used in this work were chosen in order to allow a direct comparison with other authors. For instance, the value of $5.0 \times 10^{-14} \mathrm{gr} \mathrm{cm}^{-3}$ was used by Boss et al. (2000) to calculate uniform and gaussian clouds with both a barotropic equation of state and an Eddington approximation. The value of $5.0 \times 10^{-12} \mathrm{gr} \mathrm{cm}^{-3}$ was used by Burkert \& Bodenheimer $(1993,1996)$, and also by Kitsionas \& Whitworth (2002).

The isothermal evolution of a gravitationally collapsing cloud was studied by Masunaga \& Inutsuka (1999) for determining the critical density at which the isothermal evolution is terminated. They classified the condition for which isothermality is broken down into three cases, i.e., when (1) the compressional 
heating rate overtakes the thermal colling rate; (2) the optical depth for thermal radiation reaches unity; and (3) the compressional heating rate becomes comparable with the radiative diffusion energy transport rate. The values obtained by Masunaga \& Inutsuka (1999) depend upon the temperature and opacity of the gas during the isothermal regime and the density structure of the collapsing cloud for which they used the Larson (1969) and Penston (1969) spherical isothermal similarity solution. Despite the differences between Masunaga \& Inutsuka (1999) and this calculation, for example in the assumed density structure of the collapsing cloud, there is a good agreement in general with the $\rho_{\text {crit }}$ values we have selected, see their Sect. 2 and Fig. 1.

As shown in Table 1, for models A0, A1, A2 and A3 $\rho_{\text {crit }}=5.0 \times 10^{-14} \mathrm{gr} \mathrm{cm}^{-3}$, while for models B0, B1, B2 and B3 $\rho_{\text {crit }}=5.0 \times 10^{-12} \mathrm{gr} \mathrm{cm}^{-3}$, see Sect. 2.5 for details of the initial configurations and models.

We would like to ensure the correctness of our collapse results for the Plummer cloud in this barotropic approximation by means of comparing it with a model where all the thermal physics have been accounted for. But as far as we know, the collapse of a centrally condensed cloud with all the radiation physics included has not been calculated. Therefore, we have to content ourselves at this time with the extrapolation assumed in this paper, which is that the barotropic equation of state is as good an approximation for centrally condensed clouds as it was for the uniform density case. Apart from the intrinsic difficulty of including in $\mathrm{SPH}$ a proper radiation transport treatment, there is the additional problem that the calculations become prohibitively expensive from the computational point of view.

\subsection{Initial energies}

For all the initial configurations and models (see Sect. 2.5), the particle distribution is constructed in a way that the cloud has the same ratio of thermal and rotational energy with respect to the gravitational energy, denoted by $\alpha_{\mathrm{c}}$ and $\beta_{\mathrm{c}}$, respectively. We have chosen the initial sound speed $c_{0}$ and the initial angular velocity $\Omega_{\mathrm{c}}$ of the cloud (see Table 1 ) in such a way that the cloud's energy ratios have the following numerical values:

$$
\begin{aligned}
& \alpha_{\mathrm{c}} \equiv \frac{E_{\text {therm }}}{\left|E_{\text {grav }}\right|}=0.26, \\
& \beta_{\mathrm{c}} \equiv \frac{E_{\mathrm{rot}}}{\left|E_{\text {grav }}\right|}=0.16 .
\end{aligned}
$$

These $\alpha_{\mathrm{c}}$ and $\beta_{\mathrm{c}}$ values were chosen to allow a direct comparison with other authors, see for example Bodenheimer et al. (2000). For our models, the $\beta_{\mathrm{c}}=0.16$ value gives a cloud's angular velocity $\Omega_{\mathrm{c}} \sim(2.22-5.80) \times 10^{-13} \mathrm{~s}^{-1}$, see Table 1 . For the uniform density standard isothermal test case, $\alpha_{\mathrm{c}}=0.25$ and $\beta_{\mathrm{c}}=0.20$ that gives $\Omega_{\mathrm{c}}=1.56 \times 10^{-12} \mathrm{~s}^{-1}$ (Boss \& Bodenheimer 1979; Sigalotti \& Klapp 1997), which is an order of magnitude higher than our $\beta_{\mathrm{c}}$ range values.

On the other hand, observations (Goodman et al. 1993) have shown that dense cores have velocity gradients of about 0.3 to $4.0 \mathrm{~km} \mathrm{~s}^{-1} \mathrm{pc}^{-1}$, which translates into $\Omega_{\mathrm{c}} \sim 9.6 \times 10^{-15} \mathrm{~s}^{-1}$ to $\sim 1.2 \times 10^{-13} \mathrm{~s}^{-1}$, which is slightly below our value.

The observational angular momentum - radius relation in molecular cores has been reported by Goodman et al. (1993), see their Fig. 13. Indeed, this plot has been reproduced and improved by Bodenheimer (1995) in his Fig. 1. According to these plots, for our model A3 with $R_{\mathrm{c}}=0.097 \mathrm{pc}$ its associated angular momentum is $j=6.3 \times 10^{21} \mathrm{~cm}^{2} \mathrm{~s}^{-1}$, which translate into angular velocity becomes $\Omega=1.75 \times 10^{-13} \mathrm{~s}^{-1}$, which is very close to our model A3 value.
Hence, our angular velocity range is in the happy mean of the one used by other authors in numerical calculations and suggested by observations.

\subsection{Initial velocities and mass perturbations}

For all models in this paper the initial protostellar cloud is in counterclockwise rigid body rotation around the $z$ axis; therefore the initial velocity for the $i$ th SPH particle is given by

$\boldsymbol{v}_{i}=\boldsymbol{\Omega}_{\mathbf{c}} \times \boldsymbol{r}_{i}=\left(-\Omega_{\mathrm{c}} y_{i}, \Omega_{\mathrm{c}} x_{i}, 0\right)$,

where the magnitude of the angular velocity $\Omega_{\mathrm{c}}$ has been chosen according to the model under consideration to satisfy the $\beta_{\mathrm{c}}$ value given in Eq. (5).

The initial density perturbation is applied to the mass of each SPH simulation particle $m_{i}$ regardless of the cloud model according to:

$m_{i}=m_{0}\left(1+a \cos \left(m \phi_{i}\right)\right)$

where $m_{0}$ is the unperturbed mass of the SPH simulation particle, and we set the perturbation amplitude to $a=0.1$, while the mode is fixed to $m=2$. These values have been chosen as it is customary in this field of work, see for instance Burkert \& Bodenheimer (1993), Burkert \& Bodenheimer (1996) and Sigalotti \& Klapp (2001).

\subsection{The initial models}

The initial structure of the cloud is constructed with the chosen $\alpha_{\mathrm{c}}=0.26$ and $\beta_{\mathrm{c}}=0.16$ values as given in Sect. 2.3. The assumed Plummer density profile depends upon the values selected for the three model parameters, which are the central density $\rho_{0}=3.0 \times 10^{-18} \mathrm{gr} \mathrm{cm}^{-3}$, the radius $R_{0}=0.026 \mathrm{pc}$ and the exponent $\eta=4$, see Eqs. (1) and (2). These three parameters are the same for all models. We constructed four initial configurations IC0, IC1, IC2 and IC3 with different envelopes that extend to a cloud's radius $R_{\mathrm{c}} / R_{0}=0.5,1.5,2.5$ and 3.7 , respectively. Each of the four basic initial configurations is split into two depending on the $\rho_{\text {crit }}$ value. For the models denoted by "A" (A0, $\mathrm{A} 1$, A2 and A3) $\rho_{\text {crit }}=5 \times 10^{-14} \mathrm{gm} \mathrm{cm}^{-3}$, while for the "B" models (B0, B1, B2 and B3) $\rho_{\text {crit }}=5 \times 10^{-12} \mathrm{gm} \mathrm{cm}^{-3}$. For example, the initial configuration IC1 is used for models A0 and B0, the only difference is that the collapse of the $\mathrm{B} 0$ model changes later in the evolution from isothermal to adiabatic.

As the radius and mass of the envelope increases, the lower density of the outer region gets the average density to decrease from $\rho_{\text {av }}=2.23 \times 10^{-18} \mathrm{gm} \mathrm{cm}^{-3}$ to $9.22 \times 10^{-20} \mathrm{gm} \mathrm{cm}^{-3}$ for the initial configurations IC0 and IC3, respectively, see Table 1. As the cloud's $\alpha_{\mathrm{c}}$ and $\beta_{\mathrm{c}}$ values are the same for all initial configurations, their angular velocity decreases from $\Omega_{\mathrm{c}}=5.80 \times 10^{-13} \mathrm{~s}^{-1}$ to $2.22 \times 10^{-13} \mathrm{~s}^{-1}$ for the initial configurations IC0 and IC3, respectively, see Table 1 . The third column of Table 1, labeled with $M_{\mathrm{c}}$, is the total mass contained in the cloud model.

The evolution and fragmentation of the cloud depends upon the global $\alpha_{\mathrm{c}}$ and $\beta_{\mathrm{c}}$, but also on the value of these two quantities in the central high density region that first collapses. Thus, the dynamical properties of the core are therefore of fundamental importance for determining the fate of the whole cloud's collapse.

In our models, the cloud is composed of a core that extends to $R_{0}$ plus an envelope that extends to $R_{\mathrm{c}}$, see Table 1 . For the initial configuration ICO there is no envelope and the cloud extends only to $R_{0} / 2$. The rotational and thermal energies must be shared 
Table 2. Parameters of the collapse models for the initial configurations IC.

\begin{tabular}{cccccc}
\hline \hline IC & $\alpha_{0}$ & $\beta_{0}$ & $\alpha_{0} \times \beta_{0}$ & $\alpha_{0}+\beta_{0}$ & $\Omega_{\mathrm{c}} \times 10^{-13} \mathrm{~s}^{-1}$ \\
\hline IC0 & 0.2643 & 0.1618 & 0.0428 & 0.4261 & 5.80 \\
IC1 & 0.3002 & 0.1114 & 0.0334 & 0.4116 & 3.90 \\
IC2 & 0.3350 & 0.0657 & 0.0220 & 0.4007 & 2.96 \\
IC3 & 0.3547 & 0.0374 & 0.0133 & 0.3921 & 2.22 \\
\hline
\end{tabular}

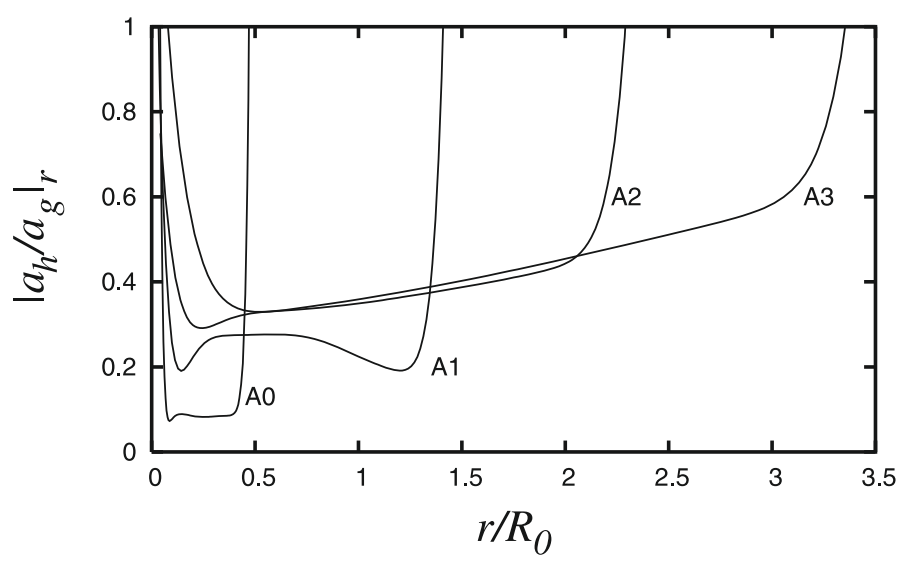

Fig. 3. Absolute value of the ratio of the hydrodynamic to the gravitational radial acceleration as a function of the radius for the four initial configurations IC0, IC1, IC2 and IC3.

somehow between the core and the envelope when constructing the whole cloud. To illustrate this energy sharing mechanism, we have calculated the energy ratios for the core alone, neglecting particles whose distance to the center of the cloud is greater than $R_{0}$. The results are presented in Table 2, where the $\alpha_{0}$ and $\beta_{0}$ values are calculated up to the core's radius $R_{0} . \Omega_{\mathrm{c}}$ is the initial angular velocity of the cloud.

According to Table 2, the dynamical properties of the core for the initial configuration ICO are, as expected, identical to the dynamical properties of the whole cloud.

Further important connections are that the bigger the envelope, the greater the thermal energy of the core while at the same time the rotational energy of the core is smaller. It is also interesting to note that for all models the sum of the energy ratios is always below 0.5 (the virial value), which means that the core has inherited the general tendency to collapse from the cloud.

The absolute value of the ratio of the hydrodynamic to the gravitational radial acceleration as a function of the radius for the four initial configurations ICO, IC1, IC2 and IC3 is presented in Fig. 3. The ratio is calculated by dividing the cloud into a number of spherical shells and averaging the radial accelerations within each shell. All initial configurations follow the same general trend, which is that near the center and surface of the cloud, the ratio reaches unity but is smaller than unity in most of the cloud. This indicates the tendency of the cloud to collapse, which is initiated in the whole cloud except very near the center and the surface. The ratio $\left|a_{h} / a_{g}\right|$ tends to unity in the center because gravity becomes very weak and there is a balance between the force due to the pressure gradient and the gravity force. In the surface the ratio also reaches unity because of the uniform initial rotation assumed for the present models. Thus, the collapse of the surface follows the collapse of central regions of the cloud. A different surface behavior could be obtained with other choices for the initial rotation, for example differential, which is something we will explore in future communications.

\subsection{Resolution}

Following Truelove et al. (1997), the resolution requirement for avoiding the growth of numerical instabilities is expressed in terms of the Jeans wavelength $\lambda_{\mathrm{J}}$, which is given by

$\lambda_{\mathrm{J}}=\sqrt{\frac{\pi c^{2}}{G \rho}}$,

where $G$ is Newton's universal gravitation constant, and $c$ is the instantaneous sound speed. To obtain a more useful form for a particle based code, the Jeans wavelength $\lambda_{\mathrm{J}}$ is written in terms of the spherical Jeans mass $M_{\mathrm{J}}$, which is defined by

$M_{\mathrm{J}} \equiv \frac{4}{3} \pi \rho\left(\frac{\lambda_{\mathrm{J}}}{2}\right)^{3}=\frac{\pi^{\frac{5}{2}}}{6} \frac{c^{3}}{\sqrt{G^{3} \rho}}$.

Nowadays it is well known that the Jeans requirement $l<\lambda_{\mathrm{J}} / 4$ (where $l$ is a characteristic length scale of the grid for a mesh based code) is a necessary condition to avoid the occurrence of artificial fragmentation. For particle based codes, Bate \& Burkert (1997) showed that there is also a mass limit resolution criterion which needs to be fulfilled besides that of Truelove et al. (1997). They showed that an SPH code produces correct results involving self-gravity as long as the minimum resolvable mass is always less than the Jeans mass $M_{\mathrm{J}}$.

Now, if we approximate the instantaneous sound speed by $c=\sqrt{p / \rho}$, then according to Eq. (3), we have that

$M_{\mathrm{J}}=\frac{\pi^{\frac{5}{2}}}{6} \frac{c_{0}^{3}}{\sqrt{G^{3} \rho}}\left[1+\gamma\left(\frac{\rho}{\rho_{\text {crit }}}\right)^{\gamma-1}\right]^{\frac{3}{2}}$.

Following Bate \& Burkert (1997), the smallest mass that an SPH calculation can resolve is $m_{r} \approx \frac{M_{\mathrm{J}}}{2 N_{\text {neigh }}}$, where $N_{\text {neigh }}$ is the number of neighboring particles included in the SPH kernel. For all models, the mass particle $m_{\mathrm{p}}$ must be such that $m_{\mathrm{p}} / m_{r}<1$.

We define the mass of the simulation particles as $m_{\mathrm{p}}=$ $M_{\mathrm{t}} / N_{\mathrm{p}}$, where $M_{\mathrm{t}}$ is the total cloud's mass, and the total number of particles $N_{\mathrm{p}}$ is 10 million for all the models in this paper.

As in Arreaga-García et al. $(2007,2008)$, because of the grid based method of implementing our initial configuration of particles, the mass of each SPH particle is such that the given radial density profile is satisfied. Despite this, we show below that for all our simulations the resolution requirement stated by Truelove et al. (1997) and Bate \& Burkert (1997) is met very well.

As an example, let us consider model A0. We follow its collapse until a peak density of $\rho_{\max }=2.10 \times 10^{-11} \mathrm{gr} \mathrm{cm}^{-3}$. For this model, from Table 1 its initial sound speed is $10620.35 \mathrm{~cm} \mathrm{~s}^{-1}$ and from Eq. (10) its minimum Jeans mass is given by $\left(M_{\mathrm{J}}\right)_{\mathrm{A} 0} \approx$ $4.0 \times 10^{32} \mathrm{gr}=0.43 M_{\odot}$, while we have $m_{\mathrm{p}}=6.28 \times$ $10^{25} \mathrm{gr}=3.15 \times 10^{-8} M_{\odot}$. Thus for model A0 we obtain the ratio $m_{\mathrm{p}} / m_{r}=5.92 \times 10^{-5}$. For model $\mathrm{B} 0$ the critical density is $\rho_{\text {crit }}=5.0 \times 10^{-12} \mathrm{gr} \mathrm{cm}^{-3}$ and the peak density is $\rho_{\max }=$ $7.42 \times 10^{-9} \mathrm{gr} \mathrm{cm}^{-3}$. In this case we obtain $M_{j}=7.56 \times 10^{30} \mathrm{gr}$ for which the mass ratio is $m_{\mathrm{p}} / m_{r}=5.89 \times 10^{-4}$. Although this ratio is a factor of 10 greater than for model $\mathrm{A} 0$, the resolution requirement for model $\mathrm{B} 0$ is also satisfied very easily.

Additionally, it was demonstrated by Nelson (2006) that for 3D - SPH simulations to accurately resolve the circumstellar disc, a resolution criterion must be fulfilled as well. This criterion establishes that for the vertical structure of the disc to be well resolved, the scale-height $H$ must be greater than four particle smoothing lengths $h$ at the disc mid-plane. As was the case 
for the Jeans criterion, for an SPH simulation this criterion is better expressed in terms of mass rather than length; Nelson then defines the Toomre mass, which is given by

$M_{\mathrm{T}}=\pi \Sigma\left(\frac{\lambda_{\mathrm{T}}}{2}\right)^{2}=\frac{\pi c^{4}}{G^{2} \Sigma}$

where $\Sigma$ is the surface density of the disc of matter and $\lambda_{\mathrm{T}}$ is the wavelength which defines the stability of the disc.

We can make a rough estimate of the Toomre mass for our simulations by making use of the following approximation. Let us divide the disc height $H$ in slices of small $\delta z$. We first calculate the average volume density $\rho_{\mathrm{av}}$ for every slice, then the relation between surface and volume densities for every slice is $\Sigma=\rho_{\mathrm{av}} \times$ $\delta z$. If we consider that $H$ for most of our models is approximately given by $H=0.02 R_{\mathrm{c}}$, where $R_{\mathrm{c}}$ is the initial radius of the cloud, then the Toomre mass for model A0 is given by $M_{\mathrm{T}}=3.56 \times$ $10^{30} \mathrm{gr}=1.79 \times 10^{-3} M_{\odot}$.

Now, following Nelson (2006), the smallest mass resolution $m_{n}$ for a simulation to prevent the appearance of numerically induced fragmentation of the disc must be given by six times the average number of the neighboring particles considered, therefore for our case we have that $m_{n} / M_{\odot} \approx\left(M_{\mathrm{T}} / M_{\odot}\right) /(6 \times 40)=$ $7.45 \times 10^{-6}$. On the other hand, as we have seen earlier, the mass of the SPH particle for our simulations is $m_{\mathrm{p}} / M_{\odot}=3.16 \times 10^{-8}$, therefore the mass ratio which is directly related to the resolution criterion is given by $m_{\mathrm{p}} / m_{n}=0.004$. Thus, our simulations also have enough mass resolution in the disc.

\subsection{Evolution code}

We have carried out the time evolution of the initial particle distribution with the Gadget 2 parallel code, which is described in detail by Springel (2005). Gadget2 is based on the tree - PM method for computing the gravitational forces and on the standard SPH method for solving the hydrodynamic Euler equations.

Gadget 2 incorporates the following standard features: (i) each particle $i$ has its own smoothing length $h_{i}$; (ii) the particles are also allowed to have individual gravitational softening lengths $\epsilon_{i}$, whose values are adjusted so that for every time step $\epsilon_{i} h_{i}$ is of order unity.

Following Gabbasov et al. (2006), some empirical formulas are known to assign a value to $\epsilon_{i}$ in order to minimize errors in the calculation of the gravitational force on a particle $i$ of the mass $m_{i}$. However, Gadget 2 fixes the value of $\epsilon_{i}$ for each timestep using the minimum smoothing length value of all particles, that is, if $h_{\min }=\min \left(h_{i}\right)$ for $i=1,2 \ldots N$, then $\epsilon_{i}=h_{\min }$.

The Gadget 2 code that we have used in this paper has implemented a Monaghan-Balsara form for the artificial viscosity, see Monaghan \& Gingold (1983) and Balsara (1995). The strength of the viscosity is regulated by setting the parameter $\alpha=0.75$ and $\beta=\frac{1}{2} \alpha$, see Eq. (14) in Springel (2005). We have fixed the Courant factor to 0.1 . In order to move the particles forward in time, Gadget 2 uses a second order leapfrog algorithm.

\section{Results}

For presenting the result of the simulations, we use iso-density contour plots for a slice of particles around the equatorial plane of the cloud. The bar located at the bottom of the plots shows the $\log _{10}$ density range at a time $t$ normalized with the initial density $\rho_{0}$. A color scale is then associated with the value of $\log _{10}\left(\frac{\rho}{\rho_{0}}\right)$. For instance, the color scale uses yellow to indicate higher densities, blue for lower densities, and green and orange for intermediate densities.

The free fall time $t_{\mathrm{ffc}}$ sets a characteristic time scale for the collapse of protostellar clouds which is given by

$$
t_{\mathrm{ffc}} \approx \sqrt{\frac{3 \pi}{32 G \rho_{0}}} .
$$

The free fall time scale $t_{\text {ffc }}$ is defined by the central density $\rho_{0}$ of the core and is the same for all models. The average density $\rho_{\mathrm{av}}$ is different for each model and decreases as the radius of the cloud increases, see Table 1 . We normalize the time history of each protostellar cloud with $t_{\mathrm{ffc}}$.

Due to the fact that the centrifugal force along the equator of the cloud is greater than at the poles, the contraction of the cloud is faster along the rotation axis, and the cloud evolves through a sequence of flat configurations.

The cloud decays in about one free fall time from a spherical to a flat configuration as most of the particles are contained in a narrow slice of matter around the equatorial plane. The accretion of particles takes place with cylindrical symmetry with respect to the rotation axis. During this first stage of gravitational contraction, the cloud reduces its dimensions to about $10 \%$ of its original size.

Later on, when the flattening of the cloud is extreme, the central part becomes slightly stretched taking the form of an oblate ellipsoid. As long as the cloud continues rotating, the central part will adopt a more elongated and slightly curved configuration, as was demonstrated by Burkert \& Hartmann (2004).

The main results of the paper are summarized as follows.

\subsection{Models $A O$ and BO: clouds with $R_{\mathrm{c}} / R_{0}=0.5$}

The initial radius of these models is only $R_{\mathrm{c}}=4.03 \times 10^{16} \mathrm{~cm}=$ $2675 \mathrm{AU} \equiv R_{0} / 2$, and the mass contained in the cloud is $M_{\mathrm{c}}=$ $0.31 M_{\odot}=0.22 M_{0}$, where $M_{0}$ is the mass in the protostellar core characterized by Eq. (2). Because of their small size, these models have the highest initial average density $\rho_{\mathrm{av}}=2.29 \times$ $10^{-18} \mathrm{gr} \mathrm{cm}^{-3}$, which is of the same order of the central density as the Plummer density function $\rho_{0}$, see Fig. 1 . These models have the highest angular velocity, $\Omega_{\mathrm{c}}=5.80 \times 10^{-13} \mathrm{~s}^{-1}$.

The density fall with the radius is not significant, i.e., the end of the cloud is reached too early for the fall of the density to be really noticeable during the evolution. For this reason, we expect the evolution of these models to be similar to the uniform density cloud model for which $\rho(r)=$ constant, see Arreaga-García et al. (2007, 2008).

When the cloud has reached its maximum flattening, the SPH particles with the highest density start forming an ellipsoidal structure in the central region of the cloud.

Burkert \& Hartmann (2004) demonstrated that as the structure collapses, it becomes even more elongated and takes a barlike shape with larger mass concentrations at the end points, which are close to or outside the foci of the initial elliptical structure. The cloud gives up some of its initial angular momentum to this resulting bar-like structure, which is large enough to prevent the total collapse of the bar. As the density of the SPH particles further increases, this bar-like structure becomes a thin filament with two density maxima located at the filament end points, as can be seen in panels c and d of Fig. 4. However, as time goes by, gas along the filament starts to be pulled in by the focal point concentrations near the ends of the filament.

Shortly after, we note that these small cores become connected by a very well defined bridge of particles; therefore for 
G. Arreaga-García et al.: Collapse of Plummer protostellar clouds
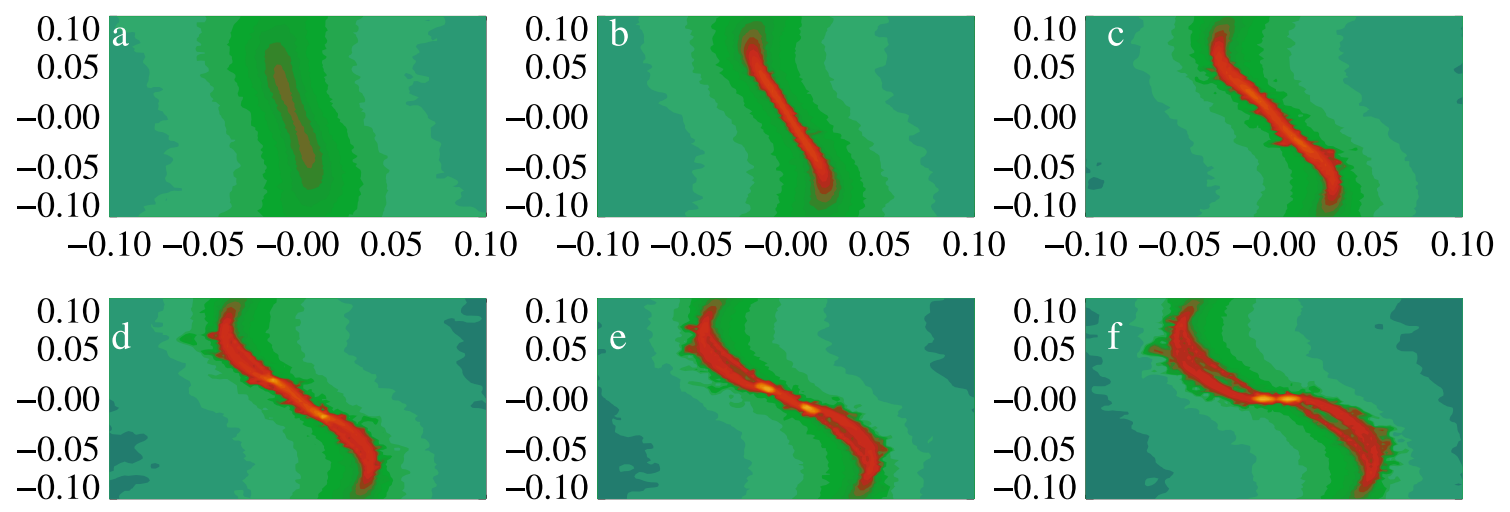

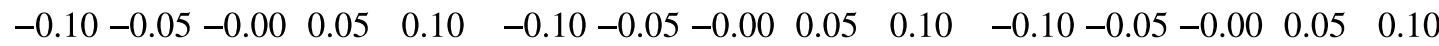
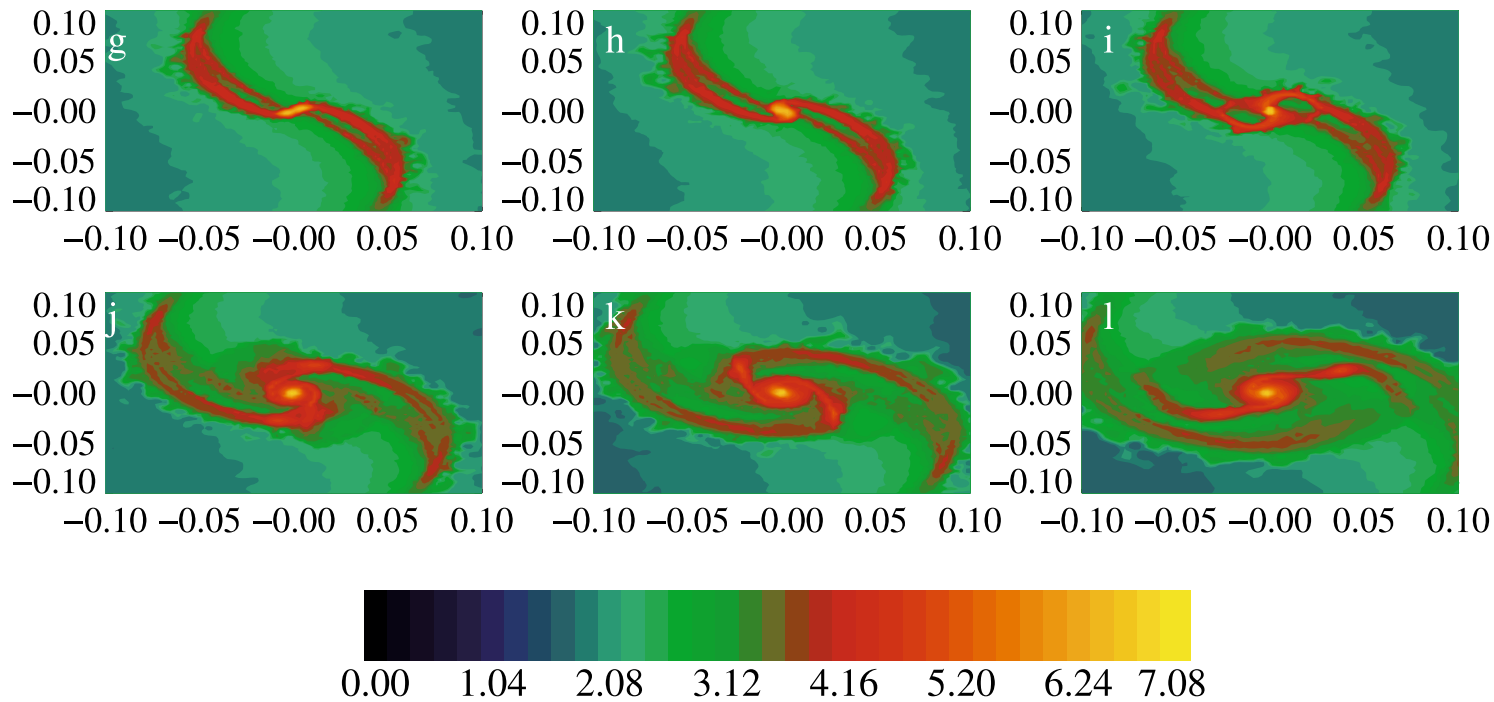

\section{$\log \rho / \rho_{0}$}

Fig. 4. Isodensity contours of the cloud mid-plane for model A0 with $\rho_{\mathrm{av}}=2.29 \times 10^{-18} \mathrm{gr} \mathrm{cm}^{-3}$ when the particle distribution reaches a peak density of a) $\rho_{\max }=9.57 \times 10^{-15} \mathrm{gr} \mathrm{cm}^{-3}$ at time $t / t_{\mathrm{ffc}}=1.3264$, b) $\rho_{\max }=2.48 \times 10^{-13} \mathrm{gr} \mathrm{cm}^{-3}$ at time $\left.t / t_{\mathrm{ffc}}=1.3468, \mathbf{c}\right) \rho_{\max }=6.22 \times 10^{-13} \mathrm{gr} \mathrm{cm}-3$ at time $t / t_{\mathrm{ffc}}=1.3632$, d) $\rho_{\max }=6.80 \times 10^{-12} \mathrm{grcm}^{-3}$ at time $t / t_{\mathrm{ffc}}=1.3735$, e) $\rho_{\max }=8.24 \times 10^{-12} \mathrm{grcm}^{-3}$ at time $t / t_{\mathrm{ffc}}=1.3812, \mathbf{f}$ ) $\rho_{\max }=1.27 \times 10^{-11} \mathrm{gr} \mathrm{cm}^{-3}$ at time $\left.t / t_{\mathrm{ffc}}=1.3904, \mathbf{g}\right) \rho_{\max }=1.23 \times 10^{-11} \mathrm{gr} \mathrm{cm}^{-3}$ at time $\left.t / t_{\mathrm{ffc}}=1.3925, \mathbf{h}\right) \rho_{\max }=1.15 \times 10^{-11} \mathrm{grcm}^{-3}$ at time $t / t_{\mathrm{ffc}}=1.3952$, (i) $\rho_{\max }=2.10 \times 10^{-11} \mathrm{grcm}^{-3}$ at time $\left.t / t_{\mathrm{ffc}}=1.4026, \mathbf{j}\right) \rho_{\max }=2.38 \times 10^{-11} \mathrm{grcm}^{-3}$ at time $t / t_{\mathrm{ffc}}=1.4241, \mathbf{k}$ ) $\rho_{\max }=2.59 \times 10^{-11} \mathrm{gr} \mathrm{cm}^{-3}$ at time $t / t_{\mathrm{ffc}}=1.4395$, and $\left.\mathbf{l}\right) \rho_{\max }=2.98 \times 10^{-11} \mathrm{gr} \mathrm{cm}^{-3}$ at time $t / t_{\mathrm{ffc}}=1.4640$.

the two models A0 and B0 a filament is formed connecting two matter clumps, regardless of the different critical density value $\rho_{\text {crit }}$ of each model. A binary system is formed which is clearly reminiscent of the uniform density model studied in ArreagaGarcía et al. (2007, 2008). Up to this point, the evolution of models $\mathrm{A} 0$ and $\mathrm{B} 0$ proceeds in an identical fashion.

The behavior of the filament of particles connecting the matter clumps is what makes the difference in the subsequent evolution: for model A0 the filament disappears, while for model B0 becomes thinner.

In model A0 the adiabatic regime enters earlier than in model B0, the collapse slows down earlier and due to gravity, the clumps become closer to one another along the filament, which finally disappears and the clumps merge to give place to a single protostellar fragment surrounded by spiral arms. However, the spiral arms start accumulating mass, where in addition to the central fragment, two exterior fragments appear resulting from the breakage of these spiral arms. Therefore, up to the dynamical time that we have been able to follow the evolution of this system, it is likely that it will end up with three fragments.
As was demonstrated by Burkert \& Hartmann (2004), every flattening gas configuration will produce a bar-like structure that forms in the cloud's central region with clumps of matter developing at each bar-like end.

The rotation of the cloud only slows down the collapse, and the bar-like structure becomes a shrinking filament. Clumps of matter continue developing, but each time over a smaller length scale.

For model B0 the collapse continues all along the filament, as can be seen in Fig. 5. The two clumps that are formed in both models tend to virialize.

\subsection{Models $A 1$ and B1: clouds with $R_{\mathrm{C}} / R_{0}=1.5$}

As can be noticed in Fig. 1, for these models the initial density profile falls to $\approx 0.1 \rho_{0}$. For this cloud the average initial density is $\rho_{\mathrm{av}}=6.95 \times 10^{-19} \mathrm{gr} \mathrm{cm}^{-3}$, the initial radius is $R_{\mathrm{c}}=1.209 \times$ $10^{17} \mathrm{~cm}=0.039 \mathrm{pc}$, and the initial angular velocity is $\Omega_{\mathrm{c}}=$ $3.90 \times 10^{-13} \mathrm{~s}^{-1}$. 

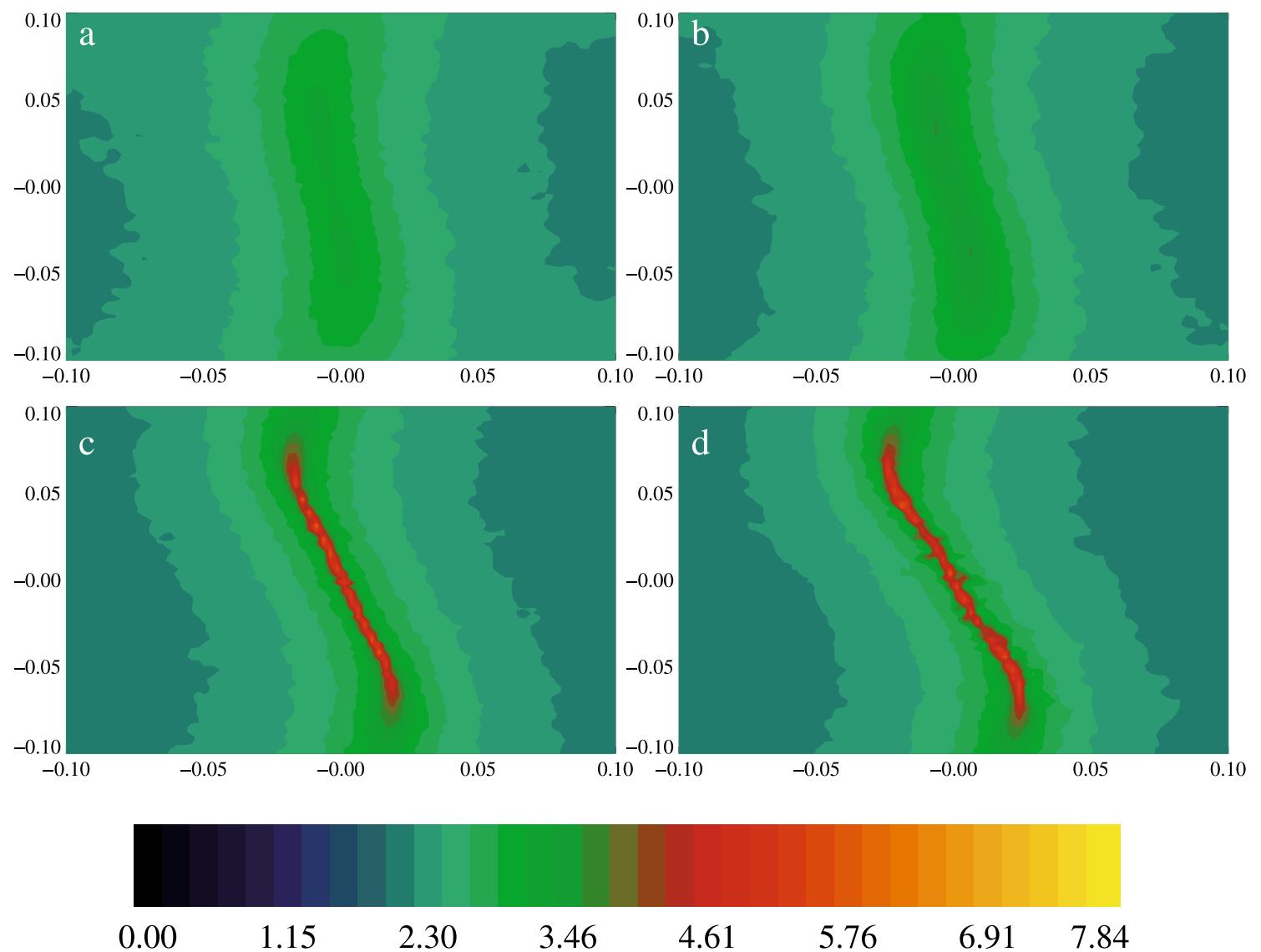

\section{$\log \rho / \rho_{0}$}

Fig. 5. Isodensity contours of the cloud mid-plane for model B0 with $\rho_{\mathrm{av}}=2.28 \times 10^{-18} \mathrm{gr} \mathrm{cm}^{-3}$ when the particle distribution reaches a peak density of a) $\rho_{\max }=4.62 \times 10^{-15} \mathrm{gr} \mathrm{cm}^{-3}$ at time $t / t_{\mathrm{ffc}}=1.3120$, b) $\rho_{\max }=1.18 \times 10^{-14} \mathrm{gr} \mathrm{cm}^{-3}$ at time $\left.t / t_{\mathrm{ffc}}=1.3222, \mathbf{c}\right) \rho_{\max }=4.99 \times 10^{-12} \mathrm{gr} \mathrm{cm}{ }^{-3}$ at time $t / t_{\mathrm{ffc}}=1.3428$, and d) $\rho_{\max }=7.41 \times 10^{-9} \mathrm{gr} \mathrm{cm}^{-3}$ at time $t / t_{\mathrm{ffc}}=1.3513$.

The fall of the density profile is more significant and the initial angular velocity is a factor of 3.2 smaller than for the models A0 and B0, and therefore we expect a different dynamical behavior.

In Fig. 6 we show the somehow complicated time history of model A1 with a critical density $\rho_{\text {crit }}=5.0 \times 10^{-14} \mathrm{grcm}^{-3}$. After the cloud flattens along the normal direction of the equatorial plane, a small bar is formed surrounded by long spiral arms. Because the rotation speed of the spiral arms and that of the bar are different, the spiral arms become separated from the bar, giving place to the appearance of two additional clumps of matter that move around the central bar. Shortly after, the central clump loses its elongated form to become cylindrical, while the two new clumps accumulate mass.

The recently formed clumps as well as the older clump develop new spiral arms. A breakage of these new spiral arms occurs, giving place to the coexistence of up to four fragments as can be seen in the last panel of Fig. 6. Until the time that we were able to follow the evolution of this simulation, the quadruple system persisted with no further merging.

In Fig. 8 (left panel) we have followed the path of the centers when only three fragments are present for a short period of time. We also show in Fig. 8 (right panel) the path of the four fragments that appears after the occurrence of the second breakage of the spiral arms.

In model B1, with $\rho_{\text {crit }}=5.0 \times 10^{-12} \mathrm{gr} \mathrm{cm}^{-3}$, the cloud experiences a stronger collapse resulting again in a system with a central bar and spiral arms, but of a smaller size than the system in model A1, as can now be seen in Fig. 7. In this case, there is no breakage of the spiral arms from the central bar. There is a tendency of the central region to fragment, retaining its spiral structure. The single resulting system shows a general tendency to virialize.

\subsection{Models $A 2$ and B2: clouds with $R_{\mathrm{C}} / R_{0}=2.5$}

In this case the initial radius of the cloud is $R_{\mathrm{c}}=2.02 \times$ $10^{17} \mathrm{~cm}=13375.0 \mathrm{AU}$, see Fig. 1 . The average initial density is $\rho_{\mathrm{av}}=2.43 \times 10^{-19} \mathrm{gr} \mathrm{cm}^{-3}$ and the initial angular velocity is $\Omega_{\mathrm{c}}=2.95 \times 10^{-13} \mathrm{~s}^{-1}$.

In model A2 a central well defined bar is formed, see Fig. 9. The surrounding spiral arms give a complete turn around the bar. Shortly after the bar decays into a single clump, we view the spirals as a ring circling the central clump. For this model we observe the formation of several fragments by means of the breakage of the spiral arms, although this phenomenon occurs in a more complicated way than in previous models. Although there 

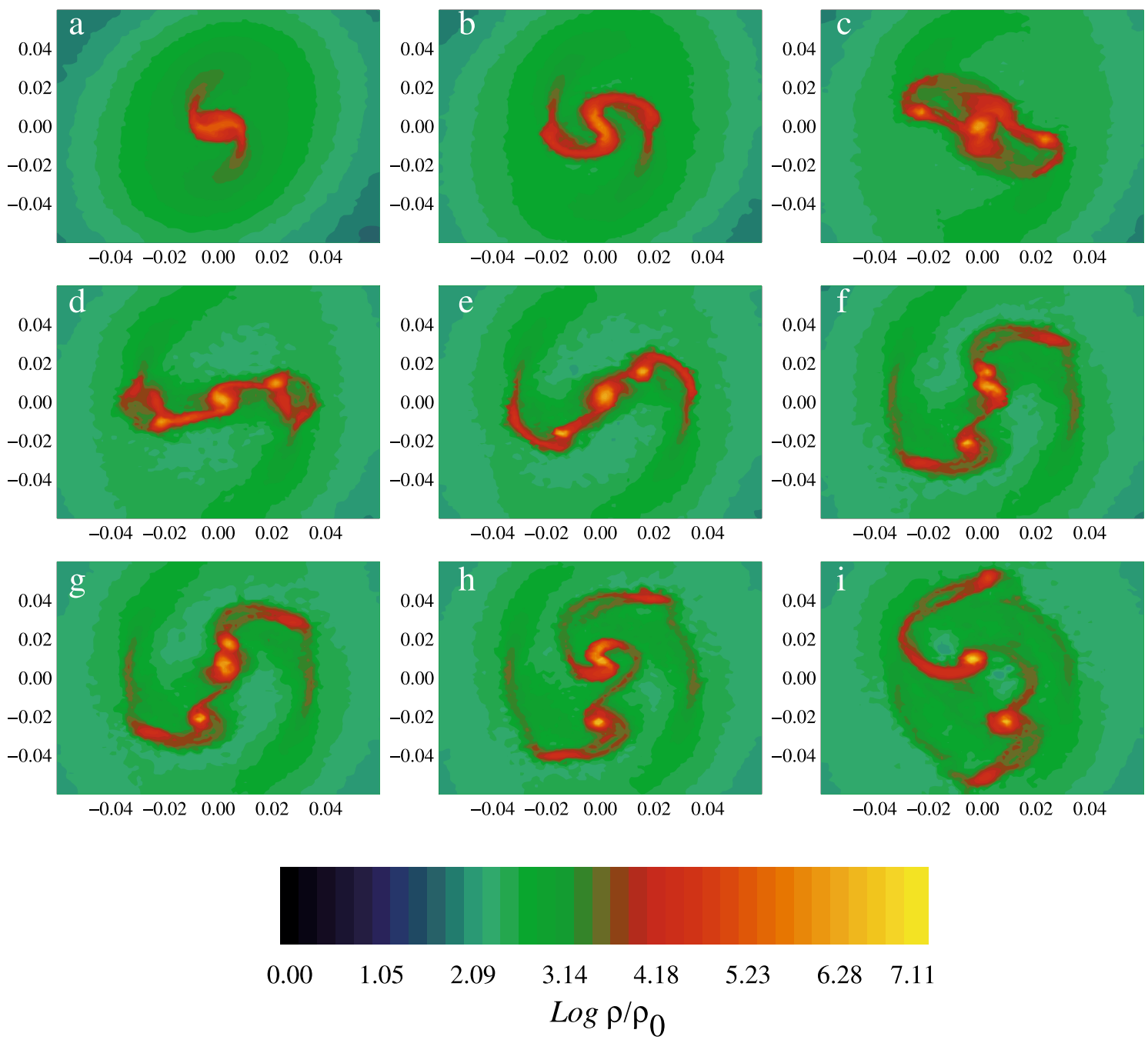

Fig. 6. Isodensity contours of the cloud mid-plane for model A1 with $\rho_{\mathrm{av}}=6.94 \times 10^{-19} \mathrm{gr} \mathrm{cm}^{-3}$ when the particle distribution reaches a peak density of a) $\rho_{\max }=6.65 \times 10^{-13} \mathrm{gr} \mathrm{cm}^{-3}$ at time $t / t_{\mathrm{ffc}}=1.4890$, b) $\rho_{\max }=2.34 \times 10^{-12} \mathrm{gr} \mathrm{cm}^{-3}$ at time $\left.t / t_{\mathrm{ffc}}=1.5422, \mathbf{c}\right) \rho_{\max }=5.96 \times 10^{-12} \mathrm{gr} \mathrm{cm} \mathrm{cm}^{-3}$ at time $t / t_{\mathrm{ffc}}=1.5954$, d) $\rho_{\max }=7.46 \times 10^{-12} \mathrm{gr} \mathrm{cm}^{-3}$ at time $t / t_{\mathrm{ffc}}=1.6167$, e) $\rho_{\max }=1.50 \times 10^{-11} \mathrm{gr} \mathrm{cm}^{-3}$ at time $t / t_{\mathrm{ffc}}=1.6273$, f) $\rho_{\mathrm{max}}=$ $1.81 \times 10^{-11} \mathrm{gr} \mathrm{cm}^{-3}$ at time $t / t_{\mathrm{ffc}}=1.6432,(\mathrm{~g}) \rho_{\max }=1.74 \times 10^{-11} \mathrm{gr} \mathrm{cm}^{-3}$ at time $\left.t / t_{\mathrm{ffc}}=1.6406, \mathbf{h}\right) \rho_{\max }=3.61 \times 10^{-11} \mathrm{grcm} \mathrm{cm}^{-3}$ at time $t / t_{\mathrm{ffc}}=1.6512$, and (i) $\rho_{\max }=3.69 \times 10^{-11} \mathrm{gr} \mathrm{cm}^{-3}$ at time $t / t_{\mathrm{ffc}}=1.6672$.

is merging among fragments, it is very likely that this simulation ends up with a binary system, see Fig. 9. The central fragment is more massive than the surrounding fragments, and some of the fragments tend to virialize.

For model B2 we see a strong collapse towards a central clump, as can be seen in Fig. 10. There is a similarity between the results of models $\mathrm{B} 1$ and $\mathrm{B} 2$, which indicates that the value of the critical density is more determinant for the result of the simulations than their slight difference in the initial density profile and the initial angular velocity.

\subsection{Models $A 3$ and B3: clouds with $R_{\mathrm{C}} / R_{0}=3.7$}

The initial radius of the cloud is $R_{\mathrm{c}}=3.0 \times 10^{17} \mathrm{~cm}=19913 \mathrm{AU}$, the average initial density is $\rho_{\mathrm{av}}=9.22 \times 10^{-20} \mathrm{gr} \mathrm{cm}^{-3}$, and the initial angular velocity is $\Omega_{\mathrm{c}}=2.22 \times 10^{-13} \mathrm{~s}^{-1}$. These $\rho_{\mathrm{av}}$ and $\Omega_{\mathrm{c}}$ values are the smallest of all models. The initial radial density profile is shown in Fig. 1. In this case, the difference in the density curves between the Gaussian and Plummer functions is more notorious than in the previous models.

In view of the outcome of the previous model B2, one would think that models A3 and B3 are not interesting, except for the fact that the central region of model A3 again shows a clear tendency to fragment towards a close binary system, as can be seen in Fig. 11.

For model B3 the collapse is so strong towards the center of the cloud that there is no indication of any fragmentation, as one can see in Fig. 12.

\section{Discussion and concluding remarks}

In order to explain the formation of binary stars, one of the mechanisms that has been proposed is prompt fragmentation, see Tohline (2002). The basic idea is that during the collapse of an isolated rotating molecular gas cloud, it may spontaneously break into two (or even more) fragments that enter into a stable orbit one around the other.

With the purpose of testing the prompt fragmentation mechanism, we present models of collapsing dense cores from centrally condensed initial states. These models are appropriate to be studied since starless cores in star-forming regions are in fact observed to be centrally condensed. The questions of how molecular clouds can reach such centrally condensed states and how the collapse is initiated are still open. 

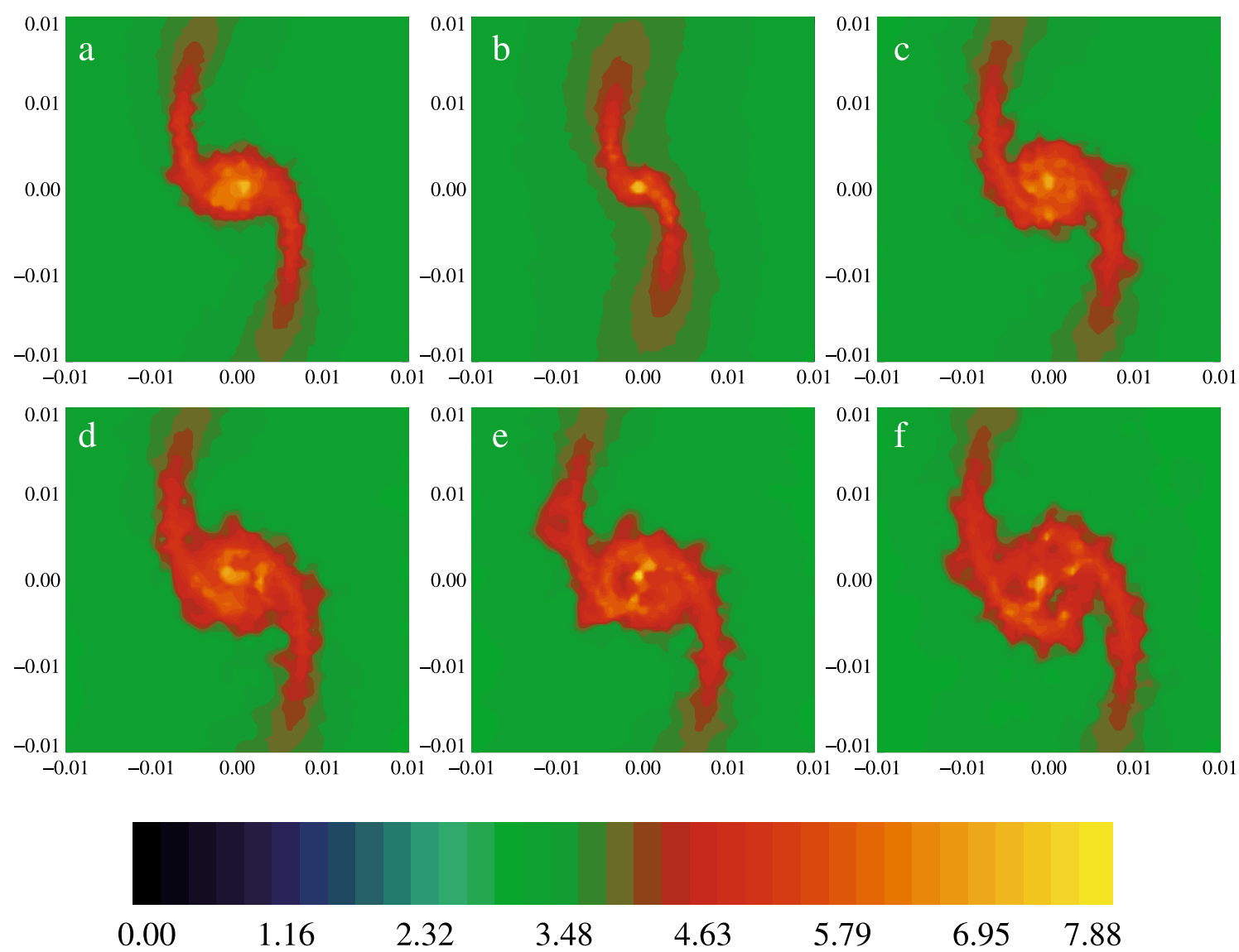

\section{$\log \rho / \rho_{0}$}

Fig. 7. Isodensity contours of the cloud mid-plane for model B1 with $\rho_{\mathrm{av}}=6.94 \times 10^{-19} \mathrm{gr} \mathrm{cm}^{-3}$ when the particle distribution reaches a peak density of a) $\rho_{\max }=7.84 \times 10^{-10} \mathrm{gr} \mathrm{cm}^{-3}$ at time $t / t_{\mathrm{ffc}}=1.4518$, b) $\rho_{\max }=3.48 \times 10^{-10} \mathrm{gr} \mathrm{cm}^{-3}$ at time $\left.t / t_{\mathrm{ffc}}=1.4624, \mathbf{c}\right) \rho_{\max }=1.10 \times 10^{-9} \mathrm{gr} \mathrm{cm}$ at time $t / t_{\mathrm{ffc}}=1.4678$, d) $\rho_{\max }=1.20 \times 10^{-9} \mathrm{gr} \mathrm{cm}^{-3}$ at time $t / t_{\mathrm{ffc}}=1.4699$, e) $\rho_{\max }=1.31 \times 10^{-9} \mathrm{grcm}^{-3}$ at time $t / t_{\mathrm{ffc}}=1.4711$, and f) $\rho_{\max }=1.35 \times 10^{-9} \mathrm{gr} \mathrm{cm}^{-3}$ at time $t / t_{\mathrm{ffc}}=1.4713$.
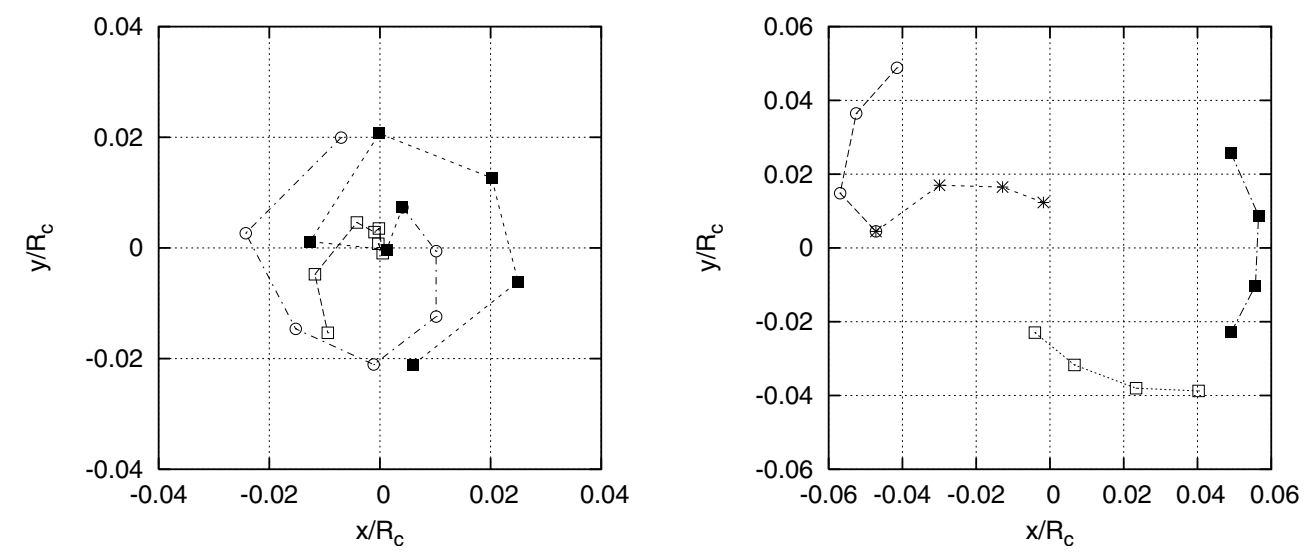

Fig. 8. Centers for model A1.

For the initially rigid rotation models presented in this paper, we have implemented a Plummer density profile with a linear mass perturbation scheme seeking to promote the generation of binary systems.

The evolution of the models studied in this work is determined by two main factors, which are the initial structure of the cloud and the value selected for the critical density $\rho_{\text {crit }}$ above which the collapse changes from isothermal to adiabatic.
The models we have presented show that as the size of the envelope increases, the tendency to fragment decreases. This is a result of the fact that as described in Sect. 2.5, for our models with bigger envelopes, $\beta_{0}, \alpha_{0}+\beta_{0}$ and $\Omega_{0}$ are lower, and the collapse that contributes to the destruction of the structure which is formed during the initial phases of the collapse is stronger. It is consequently crucial for any fragmentation to occur that there is enough rotational energy. 

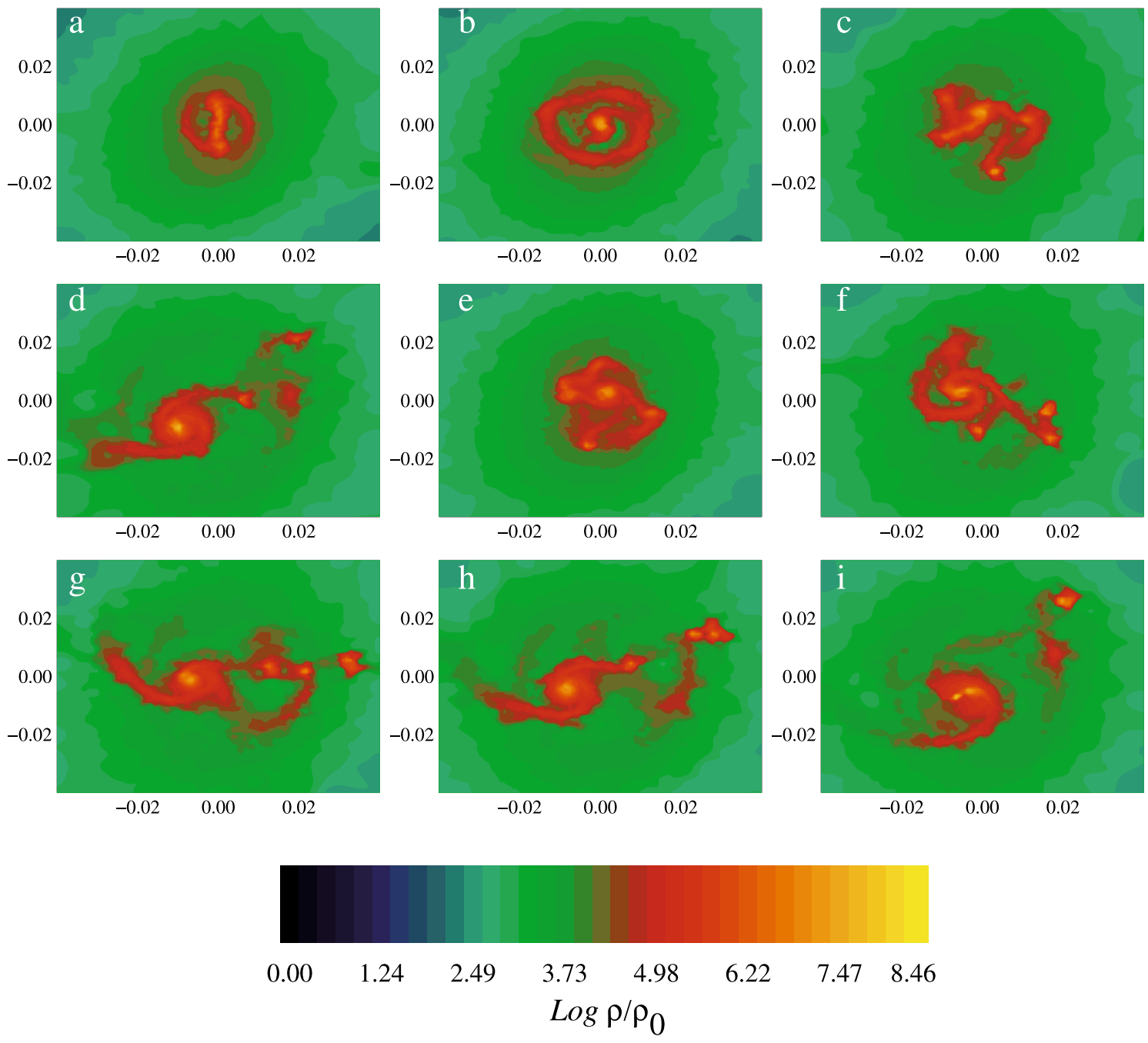

Fig. 9. Isodensity contours of the cloud mid-plane for model A2 with $\rho_{\mathrm{av}}=2.43 \times 10^{-19} \mathrm{gr} \mathrm{cm}^{-3}$ when the particle distribution reaches a peak density of a) $\rho_{\max }=2.17 \times 10^{-12} \mathrm{gr} \mathrm{cm}^{-3}$ at time $t / t_{\mathrm{ffc}}=1.5104$, b) $\rho_{\max }=8.49 \times 10^{-12} \mathrm{gr} \mathrm{cm}^{-3}$ at time $\left.t / t_{\mathrm{ffc}}=1.5562, \mathbf{c}\right) \rho_{\max }=1.17 \times 10^{-11} \mathrm{gr} \mathrm{cm}{ }^{-3}$ at time $t / t_{\mathrm{ffc}}=1.6019$, d) $\rho_{\max }=2.23 \times 10^{-11} \mathrm{gr} \mathrm{cm}^{-3}$ at time $t / t_{\mathrm{ffc}}=1.6133$, e) $\rho_{\max }=2.60 \times 10^{-11} \mathrm{gr} \mathrm{cm}^{-3}$ at time $t / t_{\mathrm{ffc}}=1.6241$, f) $\rho_{\max }=$ $3.67 \times 10^{-11} \mathrm{gr} \mathrm{cm}^{-3}$ at time $\left.t / t_{\mathrm{ffc}}=1.6340, \mathbf{g}\right) \rho_{\max }=4.42 \times 10^{-11} \mathrm{gr} \mathrm{cm}^{-3}$ at time $\left.t / t_{\mathrm{ffc}}=1.6660, \mathbf{h}\right) \rho_{\max }=5.10 \times 10^{-11} \mathrm{gr} \mathrm{cm}^{-3}$ at time $t / t_{\mathrm{ffc}}=1.6820$, and i) $\rho_{\max }=1.53 \times 10^{-10} \mathrm{gr} \mathrm{cm}^{-3}$ at time $t / t_{\mathrm{ffc}}=1.7049$.

In the case of our model A3, the rotational energy left to the core is insufficient for the collapsing core to develop any barlike structure (or S-shaped spiral pattern) in the central region. In this situation, the core can not fragment, as we have observed in our calculation.

The second factor that affects the evolution of the models studied in this work is the value of the critical density $\rho_{\text {crit }}$ for a particular model. This paper showed that if the collapse changes from isothermal to adiabatic earlier, which occurs for the models with a lower critical density $\rho_{\text {crit }}$, the collapse is slowed down more (but not completely stopped), and this gives the fragments that are generated during the collapse the possibility to survive. This can clearly be seen in Fig. 13 which shows the time evolution of the maximum density in the cloud. For the $\rho_{\text {crit }}=5.0 \times 10^{-12} \mathrm{gr} \mathrm{cm}^{-3}$ case, the collapse is not slowed down very much even up to the point that the numerical calculations are terminated, and this erased all the previously formed structure. For the models with $\rho_{\text {crit }}=5.0 \times 10^{-14} \mathrm{gr} \mathrm{cm}^{-3}$, the collapse was significantly slowed down and the structure survived. Consequently we can conclude that if the collapse changes from isothermal to adiabatic early enough, the survival probability of the fragments greatly increases.
As described in Sect. 3.1, in model A0 we observed that the two formed clumps at the filament ends merge into one single central clump of matter. This merging event occurred as a consequence of both a loss in the clump's angular momentum and the gravitational interaction between clumps.

We emphasize that there are also simulations in which the closeness between clumps also occurred, but the final merge is avoided, see for example Arreaga-García et al. (2007), Bate et al. (1995) and references in which the standard isothermal test calculation was reproduced. For this test model, the two clumps pass each other instead of merging, and they finally enter into orbit around each other.

The cause behind this different behavior, which may decide whether or not a merging occurs, is perhaps the existence of small variations in the positions and velocities of the particles, which may come from the randomness of the initial particle distribution as discussed below.

The density perturbation in Eq. (7) plays a very important role in the merging issue. Indeed, the mass perturbation will cause the development of the two small regions where the mass of the cloud will be first accreted. Once the cloud has acquired a flattened configuration, the higher density gas will form an 

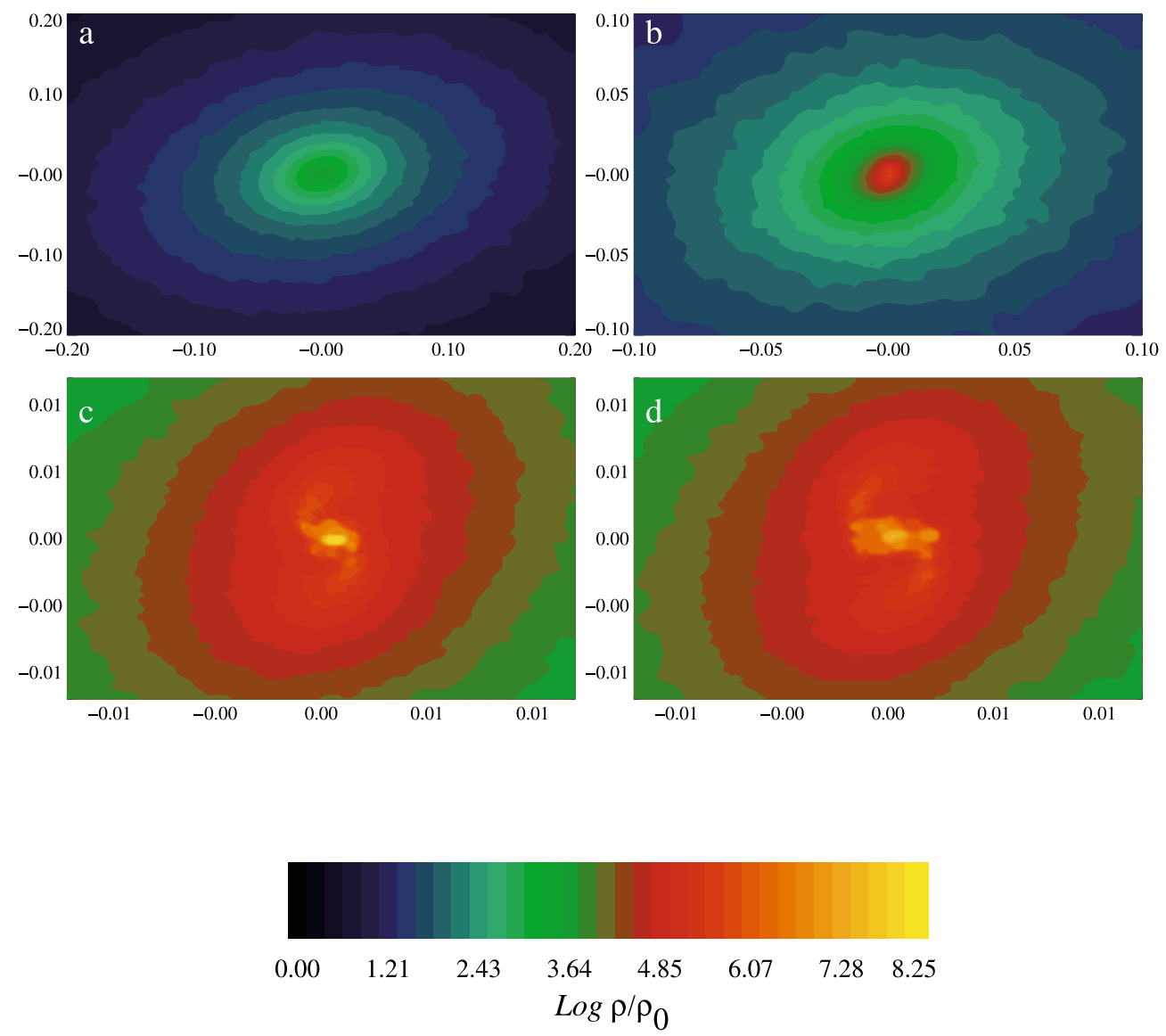

Fig. 10. Isodensity contours of the cloud mid-plane for model B 2 with $\rho_{\mathrm{av}}=2.43 \times 10^{-19}$ when the particle distribution reaches a peak density of a) $\rho_{\max }=1.31 \times 10^{-15} \mathrm{gr} \mathrm{cm}^{-3}$ at time $t / t_{\mathrm{ffc}}=1.3731$, b) $\rho_{\max }=1.08 \times 10^{-10} \mathrm{gr} \mathrm{cm}^{-3}$ at time $\left.t / t_{\mathrm{ffc}}=1.4246, \mathbf{c}\right) \rho_{\max }=2.88 \times 10^{-10} \mathrm{gr} \mathrm{cm}{ }^{-3}$ at time $t / t_{\mathrm{ffc}}=1.4303$, and d) $\rho_{\max }=5.54 \times 10^{-10} \mathrm{gr} \mathrm{cm}^{-3}$ at time $t / t_{\mathrm{ffc}}=1.4360$.

elliptical structure with these small accretion regions as focal points. As was demonstrated by Burkert \& Hartmann (2004), the fate of this elliptical structure is always to collapse into a filament with a strong accumulation of mass at each end point.

As we have implemented a symmetric mass perturbation with respect to the origin of the coordinates of the cloud's equatorial plane, the clumps that will form will be antipodes of each other in such a way that an imaginary line joining them will pass through the coordinate of origin too.

Every clump exerts a gravitational torque on the other clump. Then the velocity of the particles in the clumps begins to align with the imaginary axis of symmetry joining the clumps, that is, $\boldsymbol{v} \approx \boldsymbol{r}$, with the net effect that these particles lose angular momentum. It follows that the particles which are accreted into the clumps are those with the lower angular momentum, whereas those accreted into the surrounding spiral arms are those with the higher angular momentum. As the clumps lose their angular momentum, the gravity force which every clump exerts on the other makes them become closer to each other until they finally merge.

It is well known that when the gas gets a flattened configuration, it is susceptible to fragmentation, for instance, all clouds with $\alpha \times \beta \leq 0.12-0.15$ are expected to fragment. As far as we know, it is not yet totally clear under which physical conditions these dense cores form, become unstable and so trigger the gravitational collapse, or why all the mechanisms for fragmentation occur. This issue is as yet unclear because the parameter space of the initial conditions is very large, and there are many possibilities for the constitutive physics. Besides, several disturbing environmental effects could also be present which may affect the fate of the collapse, as discussed by Goodwin et al. (2007). However, it appears that the main mechanism for the formation of multiple systems is the fragmentation of circumstellar discs.

Goodwin et al. (2004) have considered a turbulent model for the L1544 core in which the mechanism of multiple fragmentation is illustrated. This mechanism occurs as follows. A central clump of matter is first formed, which they call the primary clump. It starts accreting mass from the surrounding gas envelope very rapidly and in a non-uniform way. Due to the bulk rotation of the flattened configuration acquired by the cloud, two spiral arms develop around the primary, which they call circumstellar accretion regions (CAR). When the CAR has accreted sufficient material, it can detach from the rotating central clump. The CAR becomes unstable and eventually fragments to produce several secondary objects.

It must be emphasized that they did not include a bulk rotation as we did in this paper, and yet we have observed a similar mechanism for the origin of multiple fragmentation in some of the models considered in this paper, for instance, models A1 and A2, which are illustrated in Figs. 6 and 9. It is interesting to mention that this fragmentation mechanism is totally suppressed when the adiabatic regime enters later in the evolution, as was the case in models B1 and B2, which can be seen in Figs. 7 and 10. Instead, for models B1 and B2 we also observed the formation of the primary clumps surrounded by short spiral 

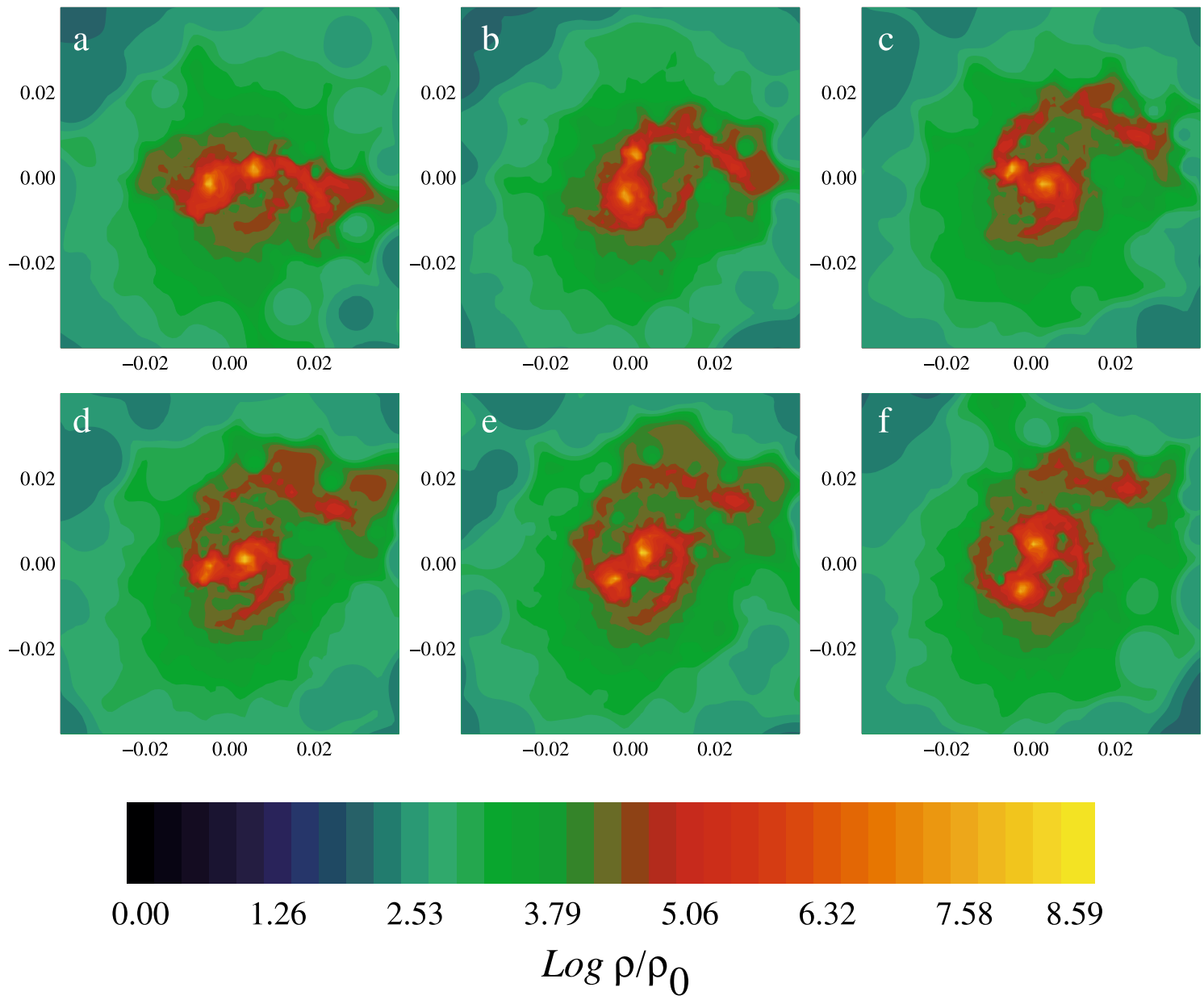

Fig. 11. Isodensity contours of the cloud mid-plane for model A3 with $\rho_{\mathrm{av}}=9.22 \times 10^{-20} \mathrm{gr} \mathrm{cm}^{-3}$ when the particle distribution reaches a peak density of a) $\rho_{\max }=4.78 \times 10^{-11} \mathrm{gr} \mathrm{cm}^{-3}$ at time $t / t_{\mathrm{ffc}}=1.6629$, b) $\rho_{\max }=5.34 \times 10^{-11} \mathrm{gr} \mathrm{cm}^{-3}$ at time $t / t_{\mathrm{ffc}}=1.6785$, c) $\rho_{\max }=5.96 \times 10^{-11} \mathrm{gr} \mathrm{cm}{ }^{-3}$ at time $t / t_{\mathrm{ffc}}=1.6941$, d) $\rho_{\max }=6.26 \times 10^{-11} \mathrm{gr} \mathrm{cm}^{-3}$ at time $t / t_{\mathrm{ffc}}=1.7014$, e) $\rho_{\max }=6.52 \times 10^{-11} \mathrm{gr} \mathrm{cm}^{-3}$ at time $t / t_{\mathrm{ffc}}=1.7066$, and f) $\rho_{\max }=6.93 \times 10^{-11} \mathrm{gr} \mathrm{cm}^{-3}$ at time $t / t_{\mathrm{ffc}}=1.7149$.

arms. Because models B1 and B2 had more time to accrete, the primary is more massive than those observed in models A1 and A2, respectively. Besides, the central clumps for models B1 and B2 show some weak tendency to develop two separate matter seeds in their centers, although it is unlikely that these two matter seeds will fragment.

We have observed that fragmentation is less favored in our models with a larger and more massive gas envelope which also have smaller initial rotation velocities. In fact, for model A3 and B3 the cloud collapses onto a single central clump. In order to promote the occurrence of fragmentation, the cloud must gain and conserve some angular momentum which will allow the spiral arms to form. But as we observed in models A3 and B3, there is not enough rotation inertia for the spiral arms to grow up, and most of the particles go directly to the central clump.

Hennebelle et al. (2004) have considered the collapse of a low-mass, rotating prestellar core which is subject to a steady increase of external pressure. They found that fragmentation is promoted when both the rotation of the cloud and the compression rate are faster. In this sense, our models with the more massive envelopes and smaller initial rotation velocities would be similar to those of Hennebelle et al. (2004) with a low compression rate.

We found it rather difficult to calculate the integral properties of a fragment in simulations where there are several transient effects. In Arreaga-García et al. (2007, 2008), the fragments showed a clear tendency to virialize. The mass of most of the resulting fragments in our simulations is very small, of the order of $\sim 0.002 M_{\odot}$.

Our main physical motivation for studying these models is to explain the behavior of the well observed L1544 dense core. Tafalla et al. (1998) have presented a multiline and continuum study of L1544, and according to them this core presents evidence to be in an advanced state of evolution toward the formation of one or two low mass stars. A visual inspection of our results presented in Sect. 3 have convinced us that it is our models A3 and B3 which could be compared with L1544.

As already discussed, the results of this paper indicate that small $\rho_{\text {crit }}$ values (heating of the gas is taken into account earlier in the collapse) can indeed result in a more structured generation even when the initial conditions might favor a very strong collapse. For models with higher critical density values, where the thermo dynamical change occurs later in the collapse, the appearance of structures is less favored. It is more likely that part of these gas structures might be converted into clumps of gas which hopefully could survive as well defined fragments, although the occurrence of merging between fragments may leave us neither with a binary nor a multiple system at all. In this sense, the Plummer models are similar to what we observed for the Gaussian models, see Arreaga-García et al. (2007, 2008), where we noted that the effect of considering the non-ideal nature of the collapse is more significant than for the uniform cloud. 

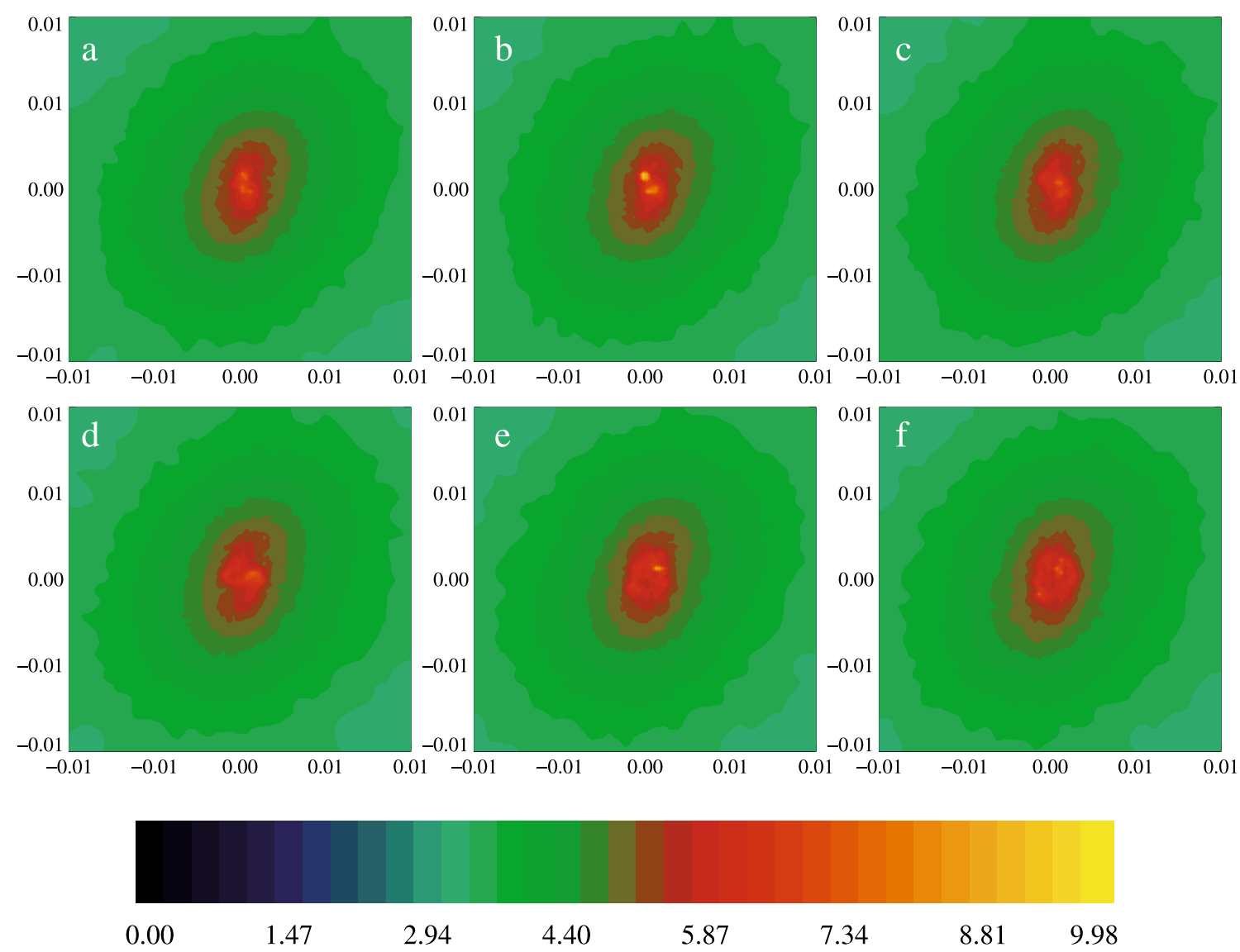

\section{$\log \rho / \rho_{0}$}

Fig. 12. Isodensity contours of the cloud mid-plane for model B 3 with $\rho_{\text {av }}=9.22 \times 10^{-20} \mathrm{gr} \mathrm{cm}^{-3}$ when the particle distribution reaches a peak density of a) $\rho_{\max }=9.52 \times 10^{-10} \mathrm{gr} \mathrm{cm}^{-3}$ at time $t / t_{\mathrm{ffc}}=1.4160$, b) $\rho_{\max }=1.51 \times 10^{-9} \mathrm{gr} \mathrm{cm}^{-3}$ at time $\left.t / t_{\mathrm{ffc}}=1.4172, \mathbf{c}\right) \rho_{\max }=2.38 \times 10^{-9} \mathrm{gr} \mathrm{cm}^{-3}$ at time $t / t_{\mathrm{ffc}}=1.4191$, d) $\rho_{\max }=1.63 \times 10^{-9} \mathrm{gr} \mathrm{cm}^{-3}$ at time $t / t_{\mathrm{ffc}}=1.4212$, e) $\rho_{\max }=2.87 \times 10^{-9} \mathrm{gr} \mathrm{cm}^{-3}$ at time $t / t_{\mathrm{ffc}}=1.4247$, and f) $\rho_{\max }=9.45 \times 10^{-9} \mathrm{gr} \mathrm{cm}^{-3}$ at time $t / t_{\mathrm{ffc}}=1.4266$.

If additional physics not included in the present calculation slow down the collapse even more, this will enhance structure formation. We will now briefly describe some of these possible physical effects.

For our present and previous calculations (Arreaga-García et al. 2007, 2008) we have followed the evolution of isolated molecular clouds. However, as the cloud collapses it may receive radiation from nearby stars or clouds. As the collapsing cloud reaches the non-isothermal regime, this incoming radiation could contribute to the heating of the gas and to further slowing down the collapse, hence favoring structure formation.

Additionally, the polarization of Zeemann measurements indicates that there are magnetic fields present in these dense cores. Thus, in a more complete theoretical calculation, magnetic fields must be taken into account. As shown by Sigalotti \& Klapp (2005), it is expected that one of the main effects of magnetic fields would be to slow down the collapse of the dense core.

There is also a technical problem which limits the amount of fragmentation that can be generated from the kind of calculations we have presented in this paper. It is just that the innermost region with the highest density and lowest free fall timescale collapses and the mass contained in the fragments is very small.
The outer regions with a much longer free fall time do not collapse very much in the timescale of the calculation. But even in the innermost region, there is only a small part of the system which collapses to very high densities. The difficulty arises from the fact that as the density of the very few fragments reaches very high values, the timestep associated with the regions occupied by these fragments becomes extremely small, and the calculation cannot be advanced any further. An interesting possibility for overcoming this difficulty is to use the sink particles approach, see for instance Jappsen et al. (2005). Using this method, a particle transforms into a sink after its density exceeds a given critical density, and thereafter nearby particles which satisfy a certain condition, fall into the sink. As this will limit the minimum timestep it may be possible to follow the further evolution of regions which are late for collapsing with this approach. The price we have to pay is to lose detailed local information of the collapse.

In recent years, there have been two approaches for the initial perturbation of the numerical model for studying the collapse and fragmentation of molecular cloud cores. The first is the one used here which assumes small linear perturbations. Other authors, for example Jappsen et al. (2005), introduce turbulent initial conditions which favor fragmentation. 


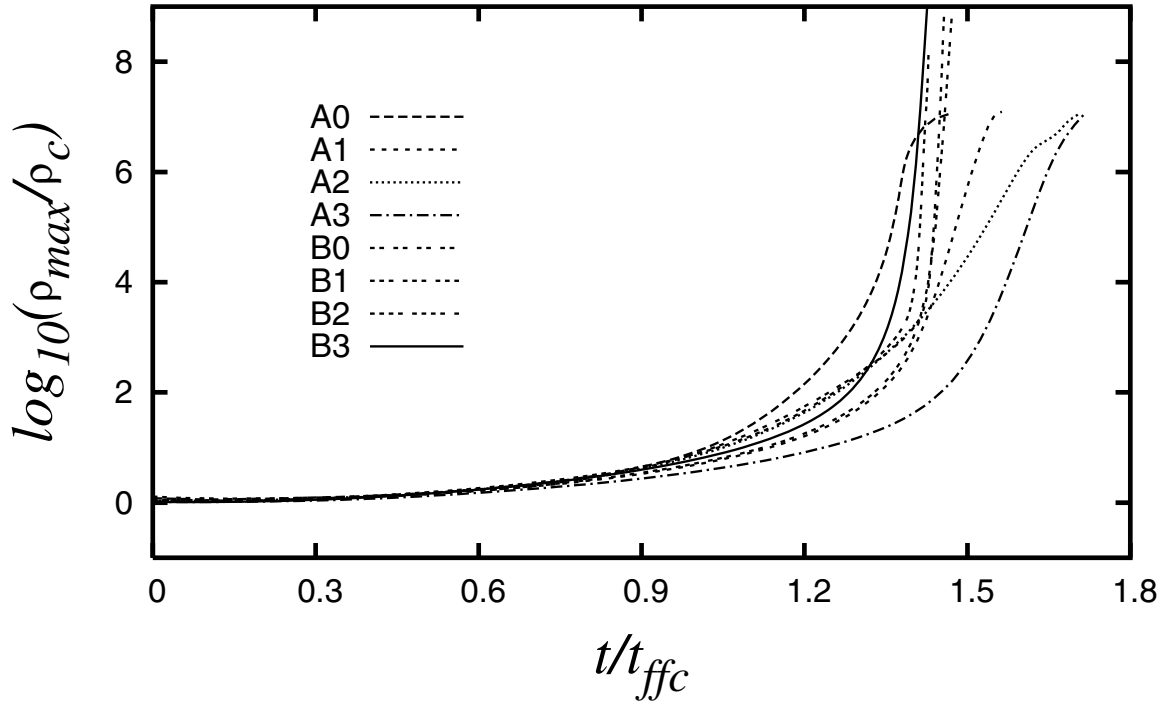

Fig. 13. Evolution of the maximum density of the cloud for all models. The time $t$ is given in units of the free fall time $t_{\mathrm{ffc}}=38460$ years, and the maximum density $\rho_{\max }$ in units of the initial central density $\rho_{0}=3.0 \times 10^{-18} \mathrm{gr} \mathrm{cm}^{-3}$.
In future communications we will consider the new physics and numerical techniques we have just described which may lead to more fragmentation.

Acknowledgements. We thank the referee Steve Stahler for providing a number of comments and suggestions that have improved the content and quality of the manuscrip. We would like to thanks ACARUS-UNISON, KanBalam-UNAM and the Instituto Nacional de Investigaciones Nucleares for the use of their computing facilities. This work has been partially supported by the Mexican Consejo Nacional de Ciencia y Tecnología (CONACYT) and the DAAD of Germany. FGR also acknowledges CONACYT and the Consejo Mexiquence de Ciencia y Tecnología (COMECYT) for a Ph.D. grant.

\section{References}

André, P., Bouwman, J., Belloche, A., \& Hennebelle, P. 2004, Ap\&SS, 292, 325 Arreaga-García, G., Klapp, J., Sigalotti, L. D., \& Gabbasov, R. 2007, ApJ, 666, 290

Arreaga-García, G., Saucedo, J., Duarte, R., \& Carmona, J. 2008, Rev. Mex. Astron. Astrofis., 44, 259

Balsara, D. 1995, J. Comput. Phys., 121, 357

Bate, M. R., \& Burkert, A. 1997, MNRAS, 288, 1060

Bate, M. R., Bonnell, I. A., \& Price, N. M. 1995, MNRAS, 277, 362

Bodenheimer, P. 1995, ARA\&A, 33, 199

Bodenheimer, P., Burkert, A., Klein, R. I., \& Boss, A. P. 2000, in Protostars and Planets IV, ed. V. G. Mannings, A. P. Boss, \& S. S. Russell (Tucson: University of Arizona Press)

Boss, A. P., \& Bodenheimer, P. 1979, ApJ, 234, 289

Boss, A. P., Fisher, R. T., Klein, R., \& McKee, C. F. 2000, ApJ, 2000, 528, 325
Burkert, A., \& Bodenheimer, P. 1993, MNRAS, 264, 798

Burkert, A., \& Bodenheimer, P. 1996, MNRAS, 280, 1190

Burkert, A., \& Hartmann, L. 2004, ApJ, 616, 288

Gabbasov, R. F., Rodriguez-Meza, M. A., Klapp, J., \& Cervantes-Cota, J. L. 2006, A\&A, 449, 1043

Goodwin, S. P., Whitworth, A. P., \& Ward-Thompson, D. 2004, A\&A, 414, 633 Goodwin, S. P., Kroupa, P., Goodman, A., \& Burkert, A. 2007, Protostars and Planets, V, 133

Goodman, A. A., Benson, P. J., Fuller, G. A., \& Myers, P. C. 1993, ApJ, 406, 528

Hennebelle, P., Whitworth, A. P., Cha, S. H., \& Goodwin, S. P. 2004, MNRAS, 348,687

Jappsen, A. K., Klessen, R. S., Larson, R. B., Li, Y., \& Mac Low, M. M. 2005, A\&A, 435, 611

Kitsionas, S., \& Whitworth, A. P. 2002, MNRAS, 330, 129

Larson, R. B. 1969, MNRAS, 145, 271

Masunaga, H., \& Inutsuka, S. 1999, ApJ, 510, 822

Monaghan, J. J., \& Gingold, R. A. 1983, J. Comput. Phys., 52, 374

Myers, P. C. 2005, ApJ, 623, 280

Nelson, A. F. 2006, MNRAS, 373, 1039

Penston, M. V. 1969, MNRAS, 144, 425

Plummer, H. C. 1911, MNRAS, 71, 460

Sigalotti, L. D., \& Klapp, J. 1997, A\&A, 319, 547

Sigalotti, L. D., \& Klapp, J. 2001, Inter. J. Mod. Phys. D, 10, 115

Sigalotti, L. D., \& Klapp, J. 2005, ApJ, 531, 1037

Springel, V. 2005, MNRAS, 364, 1105

Tafalla, M., Mardones, D., Myers, P. C., et al. 1998, ApJ, 504, 900

Tohline, J. E. 2002, ARA\&A, 40, 349

Truelove, J. K., Klein, R. I., McKee, C. F., et al. 1997, ApJ, 489, L179

Ward-Thompson, D. 2002, Science, 295, 76

Whitworth, A. P., \& Ward-Thompson, D. 2001, ApJ, 54, 317

Whitehouse, S. C., \& Bate, M. R. 2006, MNRAS, 367, 32 